\title{
Wave groupiness and spectral bandwidth as relevant parameters for the performance assessment of wave energy converters
}

\author{
Jean-Baptiste Saulnier ${ }^{\mathrm{a},{ }^{*}}$, Alain Clément ${ }^{\mathrm{a}}$, António F. de O. Falcão ${ }^{\mathrm{b}}$, Teresa Pontes $^{\mathrm{c}}$, Marc Prevosto ${ }^{\mathrm{d}}$ \\ and Pierpaolo Ricci
}

\author{
a Laboratoire de Mécanique des Fluides, Ecole Centrale de Nantes, 1, rue de la Noë, BP 92101, \\ 44321 Nantes, France \\ ${ }^{\mathrm{b}}$ IDMEC, Instituto Superior Técnico, Av. Rovisco Pais, 1, 1049-001 Lisbon, Portugal \\ ${ }^{c}$ Laboratório Nacional de Energia e Geologia, Estrada do Paço do Lumiar, 22, 1649-038 Lisbon, \\ Portugal \\ ${ }^{d}$ IFREMER, Brest Centre, DCB/ERT/HO, BP 70, 29280 Plouzané, France \\ e TECNALIA ENERGÍA, Sede de ROBOTIKER-TECNALIA, Parque Tecnológico, Ed. 202, E 48170 \\ Zamudio (Vizcaya), Spain
}

*Corresponding author : Jean-Baptiste Saulnier, email address : jean.baptiste.saulnier@ifremer.fr

\begin{abstract}
:
To date the estimation of long-term wave energy production at a given deployment site has commonly been limited to a consideration of the significant wave height $H_{\mathrm{s}}$ and mean energy period $T_{\mathrm{e}}$. This paper addresses the sensitivity of power production from wave energy converters to the wave groupiness and spectral bandwidth of sea states. Linear and non-linear systems are implemented to simulate the response of converters equipped with realistic power take-off devices in real sea states. It is shown in particular that, when the converters are not much sensitive to wave directionality, the bandwidth characteristic is appropriate to complete the set of overall wave parameters describing the sea state for the purpose of estimating wave energy production.
\end{abstract}

Keywords: Wave groupiness; Spectral bandwidth; Sea states; Wave climate; Wave energy; Wave energy converters

\section{Introduction}

The public interest for marine renewables has been increasing a lot in the recent years. Among these new ways of extracting the outstanding energy contained in seas and oceans, wave energy, in particular, has given rise to a large panel of investigation projects and new industrial activities. To date, several technologies - Wave Energy Converters (WECs) - have broken through already and are being tested worldwide. Furthermore, a certain number of public experimentation sites for mediumand full-scale units were launched in the last years, in the United Kingdom (EMEC test site in the Orkney Islands, Wave Hub in Cornwall), France (SEM-REV in Le Croisic), Ireland (Galway benign site), etc. Thus, as the developers are being more and more confronted to the sea reality, a thorough site-specific knowledge of the maritime and environmental conditions is highly desirable for several reasons. Firstly, for the own WECs' deployment and maintenance: the devices must be installed and handled preferentially during calm weather windows. Secondly, for a better evaluation of extreme conditions, where the survivability of the structure must be above all ensured. Lastly - but not 
the least reason -, for a precise prediction of performance of the WECs, which are expected to supply an optimized and significant electrical power level to the grid whatever the sea state.

Since the early years of the wave energy research up to now, the characterisation of sea states has been carried out by considering a few synthetic parameters such as the significant wave height $H_{\mathrm{s}}$ and mean period like $T_{\mathrm{z}}$ (mean zero up-crossing period), $T_{\mathrm{e}}$ (mean energy period, more common among the wave energy community), or even $T_{\mathrm{p}}$ (peak period), following the description of Hogben and Lumb (1967) through histograms and height-period scatter tables, which permit to estimate the probability of a sea state $\left(H_{\mathrm{s}}, T_{\mathrm{e}}\right)$ to be observed at a given oceanic site. Then, according to some standard sea state spectral densities like e.g. Pierson-Moskowitz (Pierson and Moskowitz, 1964) or JONSWAP (Hasselmann et al., 1973) for fully developed and growing wind-seas respectively, random waves are simulated and input into frequency/time-domain numerical models, or even generated in tank for model testing. The power figure obtained at the scale of a sea state (from one to three hours of stationary wave conditions in the open sea) is then used to build a so-called power matrix against $H_{\mathrm{s}}$ and $T_{\mathrm{e}}$, which, after cell-by-cell convolution with the corresponding joint occurrence table of the location, yields a long-term estimation of the energy extracted by the device. When the structures are sensitive to the directionality of waves, it is also frequent to include the peak or mean wave direction into the set of descriptive sea state parameters. Most of the wave climate atlases have been created following this description, such as the European Atlas WERATLAS (Pontes et al., 1997; Pontes, 1998), and, more specifically, refined atlases at country-scale like the French wave climate database ANEMOC (CETMEF, EDF), ONDATLAS (INETI/LNEG) for Portugal, etc.

However, a WEC - and, to a larger extent, any offshore structure - can behave very differently in two real sea states of same $H_{\mathrm{s}}$ and $T_{\mathrm{e}}$. It is known, indeed, - in particular from in situ measurements with directional buoys - that the spectral representation of the sea states may result in a large variety of shapes, from unimodal (one peak, e.g. Pierson-Moskowitz) to 
multimodal (two or more non-artefact peaks, see Guedes Soares, 1984; Kerbiriou, 2007), so that wave height and period are not sufficient by themselves to describe the sea state properly. Indeed, the latter may be seen as the result of the joint occurrence of remotely generated swells and a local - and maybe highly non-stationary - wind-sea. Unimodal shape-fixed spectral models based on two parameters such as Pierson-Moskowitz, therefore, are not always relevant to adequately represent the real contents of the wave field. Some bimodal analytical spectra also exist (e.g. Ochi and Hubble, 1976; Torsethaugen and Haver, 2004) but remain inconvenient to handle as default standard shapes because of the high number of required descriptive parameters (5-6 as a minimum). When the response of direction-sensitive structures in such sea states is assessed - especially in the case of spatial arrays -, neglecting the multimodal nature of sea states may lead to very erroneous results (Saulnier, 2009): in such cases, the multi-system description is to be envisioned (see Kerbiriou et al., 2007; Hanson and Phillips, 2001; Portilla et al., 2009). On the other hand, when the structure is weakly sensitive to wave directionality - like e.g. axi-symmetrical heaving floats -, the directional information may be disregarded since the wave energy frequency distribution only matters, so that the multimodality issue can be simplified to characterising the spectral bandwidth of the wave field (that is, the spreading of energy over wave frequencies) only. Moreover, it is known since a few decades (see Longuet-Higgins, 1957) that the bandwidth of sea states is directly related to the wave groupiness phenomenon: wave groups appear more pronounced as the spectrum becomes narrow. Thus, on the one hand - and while the raw data remain unavailable -, characterising the spectral bandwidth seems crucial to estimate better the actual performance of a WEC, and on the other hand, the wave groupiness properties have to be taken into account in the design of the power take-off $(P T O)$ device(s) equipping the whole machine, especially when the added "inertia" is significant (hydraulic circuits with high-pressure gas accumulators, flywheels, control laws, complex power electronics setups, etc.). Of course, trains of successive high waves are also expected to damage and ruin the 
structure somehow. However, the consideration of such extreme groupiness conditions is beyond the scope of this study.

The purpose of this paper is to investigate and illustrate the sensitivity of weakly direction-sensitive WECs (point absorbers) to the overall spectral bandwidth of sea states, which is characterised through a few spectral parameters drawn from literature - some of them having been especially introduced for wave groupiness studies. Linear as well as nonlinear hydrodynamic models are implemented to simulate the response of various types of WECs in both frequency and time domain, also including PTO devices of significant inertia (flywheel effect, short-term potential energy storage in a reservoir), whose sensitivity to wave groupiness at short term is also emphasized. The observations and conclusions of this study are expected to motivate the further inclusion of one or several "standard" spectral bandwidth/wave groupiness parameters as a notable refinement in spectral sea state description applied to the wave-energy field.

The paper is structured as follows. Section 1 refers to some spectral methods used to model the wave groupiness phenomenon, along with deterministic ones allowing to track groups of instantaneous wave energy in the time domain (SIWEH, empirical mode decomposition and Hilbert-Huang Transform, EMD-HHT). Both kinds of methods involve some devoted parameters, either spectral (bandwidth, narrowness, etc.) or deterministic (groupiness factors). In addition, a review of spectral bandwidth parameters found in literature is also made in order to complete the set of available parameters. Section 2 introduces the issue of the sensitivity to spectral bandwidth of a one degree-of-freedom (DOF) oscillating WEC, whose behaviour is assessed through stochastic modelling. Analytical formulae related to the performance of such a straightforward model in real sea states are derived to this end. Section 3 illustrates the sensitivity of several configurations of a linear axi-symmetrical WEC (stochastic model) to the spectral bandwidth of numerous real sea state spectra estimated from buoy measurements in two particular locations (Portugal and the North Sea). Section 4 deals 
with the sensitivity of a weakly direction-sensitive WEC (three-dimensional model of the SEAREV [Babarit, 2005], simulated in the time domain with non-linear hydrodynamics) to the spectral bandwidth of a set of sea states observed in a Californian location. Section 5 deals with the case of WECs equipped with inertial PTO devices, as a flywheel system (air turbine) and short-term energy-storing reservoir with nominal output power (hydraulic machinery). In both cases, the induced PTO inertia is controllable. The sensitivity to wave groupiness and spectral bandwidth is observed for both models. Section 6 eventually draws some conclusions about the consideration of the spectral bandwidth in a standard sea state description applied to wave-energy studies, and beyond, to offshore engineering.

\section{$1 \quad$ Wave groupiness properties and spectral bandwidth}

A wave group is commonly defined as a wave sequence within a record whose characteristics - height, energy, period... - exceed a given threshold value (Fig. 1). In most of the spectral methods proposed in offshore and coastal engineering literature, wave groups have been characterised as trains of successive zero up-crossing wave heights (crest-totrough). Deterministic methods have rather been based on the concept of instantaneous energy, allowing to track time sequences of significant energy level which are considered as wave energy groups. These methods, along with the spectral parameters and groupiness factors they involve, are successively presented here below.

Fig 1 Sequence of two wave groups whose heights are superior to $1 \mathrm{~m}$ in a wave record

\subsection{Spectral methods and bandwidth parameters}




\subsubsection{Sea state spectral representation}

The spectral methods are based on the classical spectral description of sea states. The ocean water elevation is assumed as a Gaussian zero-mean process, whose variance spectral density (or wave spectrum) $S(f)\left(\mathrm{m}^{2} / \mathrm{Hz}\right)$ may be estimated from in situ measurements with e.g. buoys, probes, lasers, etc. From the spectral estimation $E(f)$ of $S(f)$, or directly $S(f)$ if the wave spectra are computed by means of hindcast numerical models, the spectral moments are obtained as $(E(f)$ is kept here as default notation for wave spectra)

$m_{n}=\int_{0}^{\infty} f^{n} E(f) d f$

which in turn permit to calculate some spectral wave parameters such as the significant wave height $H_{\mathrm{m} 0}\left(\equiv H_{\mathrm{s}}\right)$, as

$H_{m 0}=4 \sqrt{m_{0}}$

and mean energy period $T_{-10}\left(\equiv T_{\mathrm{e}}\right)$, as

$$
T_{-10}=\frac{m_{-1}}{m_{0}}
$$

The spectral description of sea states is useful to calculate wave parameters from the spectral estimates of the water elevation without the need of carrying out wave-by-wave analysis. In the following, symbols $H_{\mathrm{m} 0}$ and $T_{-10}$ will be systematically used instead of $H_{\mathrm{s}}$ and $T_{\mathrm{e}}$ since they are both calculated from spectral data in this study. 


\subsubsection{Spectral methods and parameters for wave group statistics}

To establish a simple set of statistics on wave group length, Goda (1976) started from the basic assumption that successive wave heights are not correlated, which is asymptotically true when the bandwidth of the wave process is very broad. In reality however, successive waves are not absolutely uncorrelated. This point was notably emphasized by Sawnhey (1963) and Rye (1974). Indeed, Goda observed from field measurements that the wave groupiness is more pronounced as the wave spectrum becomes narrow. The peakedness factor $Q_{\mathrm{p}}$, calculated as

$Q_{p}=\frac{2}{m_{0}^{2}} \int_{0}^{\infty} f E^{2}(f) d f$

was introduced by him to characterise the groupiness level. Goda showed - along with Ewing (1973) - that the mean length of runs of successive wave heights was directly related to the value of $Q_{\mathrm{p}}$. This factor increases as the bandwidth becomes narrow: fully developed windseas typically have $Q_{\mathrm{p}}$ values close to 2 , whereas narrow-banded swells may sometimes reach higher values $(>4)$.

To take the dependence of successive wave heights into consideration, Kimura (1980) proposed a wave group theory based on a $1^{\text {st }}$-order Markov chain. The resulting statistics involve a correlation parameter $\kappa$, for which Battjes and van Vledder (1984) gave - from previous works of Arhan and Ezraty (1978) in the case of narrow-banded processes - the following spectral formulation 
$\kappa=\kappa(\tau)=\frac{1}{m_{0}} \cdot\left|\int_{0}^{\infty} E(f) \cdot e^{i 2 \pi f \tau} d f\right|$

where $\eta$ denotes the mean time lag separating two successive waves, usually taken equal to the spectral mean zero up-crossing period $T_{02}\left(=\left(m_{0} / m_{2}\right)^{1 / 2} \equiv T_{\mathrm{z}}\right)$. The correlation increases as the spectral bandwidth decreases.

From the works of Rice (1944-45) on narrow-banded random Gaussian processes, Longuet-Higgins $(1957,1984)$, among others, derived wave group statistics by considering the Hilbert envelope of the water elevation instead of wave heights. These statistics involve the narrowness parameter $v$, calculated as

$v=\varepsilon_{2}=\sqrt{\frac{m_{0} m_{2}}{m_{1}{ }^{2}}-1}$

which accounts for the bandwidth of the sea state process. Because of the presence of the $2^{\text {nd }}-$ order moment, such a parameter is somewhat sensitive to the high-frequency contents of the spectrum. Narrow-banded sea states typically have small values of $v,-$ provided the spectra have been appropriately filtered beforehand, - and therefore exhibit significant groups of waves. The narrowness parameter is denoted by $\varepsilon_{2}$ in the following, with or without filtering. Many authors (e.g. Nolte and Hsu, 1972; Medina and Hudspeth, 1990), investigated the spectrum related to the (Hilbert) envelope of the water elevation. Prevosto (1988) defined a bandwidth parameter $B_{\mathrm{w}}$ calculated as

$$
B_{w}=\frac{4}{m_{0}{ }^{2}} \int_{0}^{\infty} E^{2}(f)\left(f-\frac{m_{1}}{m_{0}}\right)^{2} d f
$$


which permits to define the shape of an ideal envelope spectrum. Let us stress the specificity of this parameter (in $\mathrm{Hz}$ ), which does not depend on the frequency location of the spectrum.

\subsubsection{Bandwidth parameters from literature}

Out of the frame of studies especially devoted to wave groupiness, several authors have proposed spectral bandwidth parameters for other purposes. In Mollison (1985) for instance, a proposal of standard wave parameters for a more complete description of the wave climate for the wave energy exploitation was presented. The broadness parameter $\varepsilon_{0}$, in particular, is introduced to characterise the spectral bandwidth of sea states. It is defined as the relative standard deviation of the period wave spectrum $\left(E(T)=E(1 / T=f) / T^{2}\right)$ and therefore expressed as

$$
\varepsilon_{0}=\sqrt{\frac{m_{0} m_{-2}}{m_{-1}^{2}}-1}
$$

Very similar to $\varepsilon_{2}$ in the formulation, this parameter is very much less sensitive to high frequency components due to the presence of low-order moments.

Smith et al. (2006) computed a new bandwidth parameter - similar to $\varepsilon_{2}$ and $\varepsilon_{0}$ - from a preliminary study led by Woolf (2002). This parameter, symbolized here by $\varepsilon_{1}$, is expressed as

$$
\varepsilon_{1}=\sqrt{\frac{m_{1} m_{-1}}{m_{0}^{2}}-1}
$$


Likewise, such a parameter is expected to be less sensitive to the high frequency contents of sea states than $\varepsilon_{2}$.

Medina and Hudspeth (1987) introduced the factor $Q_{\mathrm{e}}$ - very similar to Goda's $Q_{\mathrm{p}}$ - to control the variance variability of numerically simulated wave processes. Its expression is

$$
Q_{e}=\frac{2 m_{1}}{m_{0}^{3}} \int_{0}^{\infty} E^{2}(f) d f
$$

They indicate that $Q_{\mathrm{e}}$ is related to the inverse of the equivalent spectral bandwidth introduced by Blackman and Tukey (1959), here denoted by $\Lambda(\mathrm{Hz})$ and calculated as

$$
\Lambda=\frac{\left(\int_{0}^{\infty} E(f) d f\right)^{2}}{\int_{0}^{\infty} E^{2}(f) d f}=\frac{m_{0}^{2}}{\int_{0}^{\infty} E^{2}(f) d f}
$$

Indeed, it can be easily shown that $\Lambda=2\left(\varepsilon_{1}^{2}+1\right) /\left(T_{-10} Q_{\mathrm{e}}\right)$.

\subsection{Deterministic methods and groupiness factors}

\subsubsection{Smoothed Instantaneous Wave Energy History}

The Smoothed Instantaneous Wave Energy History (SIWEH) was defined by Funke and Mansard (1980) to identify and control the wave groupiness in time series of water

elevation for the generation of grouped waves in tank. The SIWEH $\left(\mathrm{m}^{2}\right)$ is computed as 
$\operatorname{SIWEH}(t)=\frac{1}{T_{p}} \int_{-\infty}^{\infty} \eta^{2}(t+\tau) Q(\tau) d \tau$

where $Q(\eta)$ is the Bartlett window

$Q(\tau)=\left\{\begin{array}{cc}1-\frac{\tau}{T_{p}}, & |\tau|<T_{p} \\ 0, & |\tau| \geq T_{p}\end{array}\right.$

The level of groupiness in a given record is then characterised by the groupiness factor $G F_{\text {SIWEH, defined as }}$

$$
G F_{S I W E H}=\frac{\sigma_{S I W E H}}{S I W E H(t)}
$$

where the bar denotes the time average and $\zeta_{\text {SIWEH }}$ denotes the standard deviation of $\operatorname{SIWEH}(t)$. For a given sea state with given target spectrum, it can be shown that the mean value of the SIWEH is $m_{0}$, so that $G F_{\text {SIWEH }}$ accounts for the relative variability of the energy signal in time. If this factor is close to 1 , the groupiness is high; as $G F_{\text {SIWEH }}$ tends to 0 , the wave signal tends to a sine wave. For the sake of exhaustiveness, let us evoke a possible alternative method for the calculation of the groupiness factor, which was introduced by List (1991). The new factor is calculated from the low-pass filtering of the water elevation modulus $|\eta(t)|$ and therefore requires the definition of an appropriate cut-off frequency. Yet, it is not included in this study. 
The Empirical Mode Decomposition and Hilbert-Huang Transform (EMD-HHT) method was proposed by Huang et al. (1998) as an alternative to the classical Fourier decomposition, especially when the observed phenomena are not stationary nor linear, just as waves are by nature. The EMD consists in decomposing - by sifting process - the signal into a finite number of narrow-banded signals $c_{\mathrm{j}}(t)$ called Intrinsic Mode Functions (IMF), which characterise different time scales of the phenomenon up to a residue (long period and small amplitude trend, out of the scope of the analysis), and whose instantaneous frequency $\omega_{\mathrm{j}}(t)$ and amplitude $a_{\mathrm{j}}(t)$ can be properly defined (Fig. 2). The Hilbert spectrum $H(\omega, t)$ represents the distribution of the amplitude of each IMF against frequency and time. By integrating the spectrum against frequencies, the instantaneous energy signal $I E(t)\left(\mathrm{m}^{2}\right)$ is here calculated as

$$
I E(t)=\sum_{j} H^{2}\left(\omega_{j}(t), t\right)
$$

Similarly to the SIWEH, the groupiness factor $G F_{\text {IE }}$ may be formed as the ratio

$$
G F_{I E}=\frac{\sigma_{I E}}{I E(t)}
$$

where $\sigma_{\mathrm{IE}}$ denotes the standard deviation of $I E(t)$. Let us mention that a similar groupiness factor may be found in Dong et al. (2008), which is derived from the wavelet energy density of the wave signal instead of $I E(t)$.

Fig. 2 Extract of IMF (top) derived by EMD-HHT from a wave signal, with related instantaneous frequency (middle) and amplitude (bottom) 


\section{First approach: Stochastic modelling of a 1-DOF heaving axi-symmetrical buoy}

\subsection{WEC Principle}

The simplest WEC model is a one degree of freedom (DOF) linear WEC oscillating in heave thanks to the wave action, and rigidly linked to the sea bottom (Fig. 3). It is excited by the incident waves $\eta(t)$ - whose amplitudes are small enough with respect to wavelength to satisfy the linear theory conditions. The energy absorption is performed through a linear damper $\left(C_{\text {PTO }}\right)$ so that the whole system may be seen as a linear filter. In addition, the WEC is assumed as punctual (point absorber) and axi-symmetrical with respect to the vertical axis (orthogonal to the waterplane at rest): accordingly, it is supposed to be absolutely insensitive to wave directionality.

Fig. 3 Physical sketch of the one degree-of-freedom WEC oscillating in heave

In Figure 3, O denotes the origin of the space coordinate system (Oxyz), with $z=0$ corresponding to the waterplane at rest, and $\mathrm{G}$ is the float's centre of gravity.

\subsection{Frequency domain modelling of the WEC}

The mechanical equation against the vertical axis $(\mathrm{O} z)$ (with positive $z$ going upwards) in time domain may be written as

$$
m \ddot{z}(t)=F_{e}(t)+F_{r}(t)+F_{h}(t)+F_{\text {PTO }}(t)
$$


where $m$ is the float's mass, $F_{\mathrm{e}}(t)$ the vertical wave excitation force exerted on the float, $F_{\mathrm{r}}(t)$ the vertical radiation force (i.e. the force exerted by the hull on the fluid when oscillating), $F_{\mathrm{h}}(t)$ the hydrostatic restoring force in the sea-water (relative to the still water level), and $F_{\text {PTO }}(t)$ the vertical effort of the PTO device (here, a pure damper) on the float. As each of these forces is linear with respect to the float's vertical motions, if one considers a harmonic excitation of circular frequency $\omega(=2 \pi f)$, equation (17) in the frequency domain simplifies to

$$
\underline{z}(\omega)=\underline{H}_{z \eta}(\omega) \cdot \underline{\eta}(\omega)
$$

where $\underline{z}(\omega)$ is the complex amplitude of the float's vertical motion (heave), $\underline{\eta}(\omega)$ the complex amplitude of the water elevation at point $\mathrm{O}$, and $\underline{H}_{\eta \mathrm{z}}(\omega)$ the complex transfer function linking water elevation to heave motion. If one considers heave velocity $\underline{\dot{z}}(\omega)=d \underline{z} / d t=\mathrm{i} \omega \underline{z}(\omega)$ instead, the corresponding transfer function $\underline{H}_{\eta \dot{z}}(\omega)$ - called mechanical impedance - may classically be found written under the form

$$
\underline{H}_{\eta \dot{z}}(\omega)=\frac{\Phi(\omega)}{\left[B(\omega)+C_{\text {PTO }}\right]+i\left[\omega(m+A(\omega))-\frac{\rho g S}{\omega}\right]}
$$

which involves wave excitation $(\underline{\Phi}(\omega))$ and radiation coefficients $(A(\omega), B(\omega))$, see Falnes (2002), Falcão (2007). The linear PTO exerts the instantaneous force

$$
F_{P T O}(t)=-C_{P T O} \cdot \dot{z}(t)
$$

on the system. Hence, the instantaneous power absorbed by the buoy 
$P_{P T O}(t)=-F_{P T O}(t) \cdot \dot{z}(t)=C_{P T O} \cdot \dot{z}^{2}(t)$

The mean power absorbed in a monochromatic sea state characterised by harmonic $\omega$ is therefore

$\overline{P_{P T O}}=C_{P T O} \cdot \sigma_{\dot{z}}^{2}=\frac{1}{2} C_{P T O}|\underline{\underline{z}}(\omega)|^{2}$

where $\zeta_{\dot{z}}$ denotes standard deviation of heave velocity.

\subsection{Performance of the WEC in real sea states}

As said in the last section, real sea states are characterised by their variance density spectrum $E(f)$. According to general properties of linear systems, the corresponding velocity variance density is given by

$E_{\dot{z}}(f)=\left|\underline{H}_{\eta \dot{z}}(f)\right|^{2} \cdot E(f)$

Assuming the water elevation $\eta$ is a Gaussian random process, the behaviour of the buoy (heave velocity $\dot{z}$ ) in the sea state will be Gaussian too, by linearity of the mechanical system. The velocity variance in equation (22) is obtained as the $0^{\text {th }}$-order spectral moment of density $E_{\dot{z}}(f), m_{\dot{z} 0} \equiv \zeta_{\dot{z}}^{2}$. Hence, the mean power absorbed in panchromatic sea states 


$$
\begin{aligned}
\overline{P_{P T O}} & =C_{P T O} \cdot m_{\dot{z} 0} \\
& =C_{P T O} \cdot \int_{0}^{\infty} E_{\dot{z}}(f) d f \\
& =C_{P T O} \cdot \int_{0}^{\infty}\left|\underline{H}_{\eta \dot{z}}(f)\right|^{2} E(f) d f \\
& =\int_{0}^{\infty} P T F(f) E(f) d f
\end{aligned}
$$

where $\operatorname{PTF}(f)=\left.C_{\mathrm{PTO}}{ }^{*} \underline{H}_{\eta \dot{z}}(f)\right|^{2}$ is called the power transfer function of the WEC. Accordingly, the groupiness of $\dot{z}(t)$ - which influences $\zeta_{\dot{z}}^{2}$ - directly accounts for the performance of the WEC and, a fortiori, has a larger direct effect than the original groupiness of elevation $\eta(t)$. This first simple approach in the frequency domain explains why the incident wave groups may or not have an impact on the wave energy conversion according to the dynamics of the system at the scale of a sea state. For same $H_{\mathrm{m} 0}$ and $T_{-10}$, the spectral shape variability of the sea states may result in very different mean power values indeed.

\section{Sensitivity to spectral bandwidth of axi-symmetrical heaving IPS WECs}

\subsection{Stochastic modelling and WEC configurations}

Following the same modelling procedure, the behaviour of a 2-DOF axi-symmetrical WEC is assessed in real sea states. The device (IPS buoy) is depicted in Figure 4: a floating cylinder $(x)$ is rigidly connected to a submerged vertical pipe, which houses a freelyoscillating piston $(y)$; both parts are moving against each other along the same axis, which is the own buoy's and pipe's axi-symmetry axis. The dimensions of the whole machine are given in Figure 4. 
The relative motion $\delta=x-y$ (random variable of zero-mean) is converted into instantaneous energy thanks to a linear PTO of frequency-independent damping and stiffness coefficients $C_{\text {PTO }}$ and $K_{\text {PTO }}$ respectively (the PTO efforts upon the WEC here are the sum of linear damping and restoring forces in Eq. (17) indeed). Four configurations $\left(C_{\mathrm{PTO}}, K_{\mathrm{PTO}}\right)$ of the device are envisioned: IPS1 (1113480kg.s $\left.{ }^{-1}, 5.10^{3} \mathrm{~kg} \cdot \mathrm{s}^{-2}\right)$, IPS2 $\left(158177 \mathrm{~kg} . \mathrm{s}^{-1}, 5.10^{3} \mathrm{~kg} . \mathrm{s}^{-2}\right)$, IPS3 (8425926kg.s $\left.{ }^{-1}, 5.10^{3} \mathrm{~kg} . \mathrm{s}^{-2}\right)$ and IPS4 (536351 kg.s $\left.{ }^{-1}, 5.10^{5} \mathrm{~kg} . \mathrm{s}^{-2}\right)$, whose power transfer functions are shown in Figure 5 against wave period $T=1 / f$. IPS1 and IPS2 correspond to realistic mechanical sensitivities over two different period ranges, whereas IPS3 and IPS4 refer to ideal narrow and broad configurations respectively.

Fig. 5 Power transfer functions of the four linear PTO configurations against wave period

The transfer functions are computed with the aid of the boundary element method code WAMIT $^{\circledR}$ and equation (24) is used to derive the mean extracted power of each configuration in a given sea state of energy density $E(f)$. In order to characterise their performance in a general way for any sea state, the following capture width parameter (in m) is formed, as the ratio

$$
\chi_{P}=\frac{\overline{P_{P T O}}}{P_{w}}
$$

where $P_{\mathrm{w}}$ denotes the omnidirectional wave power (in $\mathrm{kW} / \mathrm{m}$ ), approached in deep water by

$$
P_{w} \approx 0.4906 H_{m 0}^{2} T_{-10}
$$


At any given depth $h, P_{\mathrm{w}}$ is otherwise calculated as

$$
P_{w}=\rho g \int_{0}^{\infty} c_{g}(f, h) E(f) d f
$$

where $c_{\mathrm{g}}(f, h)$ is the group celerity of waves, which is a function of both frequency and depth. Let us stress that parameter $\chi_{\mathrm{P}}$ is not dependent on $m_{0}$ (i.e. $H_{\mathrm{m} 0}$ ) by definition. Therefore, the only influencing characteristics are the mean wave period and the spectral bandwidth (shape). To be precised that a low performance value in the sense of $\chi_{\mathrm{P}}$ does not necessarily mean a low mean power value since the capture width is an intensive figure (ratio).

\subsection{Wave spectral data}

Two wave climates are considered in the following, the one related to the Western coast of Portugal (Figueira da Foz, " $F F ", h \sim 90 \mathrm{~m}$ ) and the other one experienced at the K13 station ("K13", $h \sim 30 \mathrm{~m}$ ), near the Dutch coasts in the North Sea (Fig. 6). Both climates are known from in situ buoy measurements carried out over a long period of time, as about 13 and 9 years for $\mathrm{FF}$ and $\mathrm{K} 13$ respectively. The omnidirectional energy spectra $E(f)$ are collected every three hours, out of stormy conditions. Both spectral samples are considered as homogeneous and composed of 26500 and 23300 spectra respectively for FF and K13 locations. FF data were provided by the Portuguese Hydrographic Institute (Instituto Hidrográfico) whereas K13 ones were ceded by the Dutch Rijkswaterstaat. The characteristics of each set of buoy data are given in Table 1 below. Let us add that unimodal 
swell- and wind-sea dominated sea states are essentially expected to occur at FF and K13 respectively.

Fig. 6 Location of the buoys deployed off Figueira da Foz (Portugal) and near the K13 platform (North Sea)

Table 1 In situ wave data: characteristics of buoy measurement campaigns carried out in Portugal (Figueira da Foz) and in the North Sea (K13 station)

\subsection{Capture width distribution and sensitivity to spectral bandwidth parameters}

\subsubsection{Capture width distribution}

Figures $7(\mathrm{a}-\mathrm{h})$ depict the distribution (mean and $90 \%$ confidence interval) of $\chi_{\mathrm{P}}$ against mean energy period $T_{-10}$ at both locations for each IPS configuration. The performance obtained using Bretschneider spectra as input sea states is also added to the plots.

Bretschneider spectra are here computed from $H_{\mathrm{m} 0}$ and $T_{\mathrm{p}}$ according to the general expression (see Saulnier, 2009)

$S_{Q p}(f)=\frac{H_{m 0}^{2}}{16} T_{p}\left(1+2 Q_{p}\right) \frac{\exp \left[-\alpha\left(f T_{p}\right)^{-2 Q_{p}}\right]}{\left(f T_{p}\right)^{1+2 Q_{p}}}$

where $Q_{\mathrm{p}}=2$ and $\alpha=1+1 / 2 Q_{\mathrm{p}}=5 / 4$. For such a spectrum (fully developed sea: $Q_{\mathrm{p}}=2$ ), it is shown that both energy and peak periods are related to each other as: $T_{-10} \sim 0.857^{*} T_{\mathrm{p}}$. 
Figures $7(\mathrm{a}-\mathrm{h})$ lead to the following observations. Firstly, the performance variability is significant, especially over particular period ranges, which correspond to the natural sensitivity band of each configuration. The variability observed at given $T_{-10}$ is clearly due to that of the sea states' spectral shape. Secondly, the general patterns of the distributions do not apparently depend on the location (FF dominated by swells in deep water and K13 dominated by wind-seas in finite depth), but rather on the WEC's transfer function. Some slight differences from a location to another may be due to the water depth conditions, which influence the computation of $P_{\mathrm{w}}$ (Eq. (26)\&(27)). Lastly, the performance estimated with ideal Bretschneider input spectra is generally overestimating the field averaged one.

\section{Fig. 7 Distribution of capture width against mean energy period obtained from long-term} wave measurements in Figueira da Foz (left) and K13 (right) for PTO configurations IPS1 to IPS4 (top to bottom); capture width obtained with Bretschneider sea states (dotted line)

These comments raise the fact that describing the sea state through the significant wave height $\left(H_{\mathrm{m} 0}\right)$ and energy period $\left(T_{-10}\right)$ only is not sufficient to characterise the performance accurately, even for very simple - linear - WEC configurations as those modelled here. Accordingly, analytical unimodal and shape-fixed wave spectra such as Bretschneider (or Pierson-Moskowitz, JONSWAP 3.3...) are not relevant to predict the actual performance of such WECs.

\subsubsection{Sensitivity to spectral bandwidth parameters}

In order to examine the relevance of characterising the spectral shape of sea states as complementary information to assess the performance of the WEC, the scatter plot of capture 
width $\chi_{\mathrm{P}}$ against some bandwidth parameters listed in Section 1 is observed. Figure 8 depicts an example for IPS1 simulated in sea states with $T_{-10}$ around $7 \mathrm{~s}$ in FF against parameter $\varepsilon_{0}$. In this figure, a clear correlation is noticed: this means that for such sea states, the performance of the device is sensitive to this third parameter, so that the actual absolute performance (Eq. (24)) may be approached from the knowledge of $H_{\mathrm{m} 0}, T_{-10}$ and $\varepsilon_{0}$. A least squares fit with quadratic trend is applied to the scatter in order to evaluate the level of correlation. The determination coefficient $R^{2}(\in[0 ; 1])$ is used as an indicator of the sensitivity to the spectral bandwidth parameter: in the present case, the value is quite high $\left(R^{2} \sim 0.88\right)$. It is calculated for each bandwidth parameter of $\varepsilon_{0}, \varepsilon_{1}, \varepsilon_{2}, Q_{\mathrm{p}}, \kappa$, and $B_{\mathrm{w}}$ for various energy period bands with a sufficient amount of spectra in each sample, never less than 50 - for each IPS configuration at FF and K13. The resulting curves are presented in Figure 9.

Fig. 8 Distribution of capture width against Mollison's relative bandwidth parameter $\varepsilon_{0}$ in $7 \mathrm{~s}$ energy period sea states in Figueira da Foz for PTO configuration IPS1; quadratic regression in the least-squares sense with determination coefficient $R^{2}$

Most of the $R^{2}$ curves are oscillating: they reach high values over some particular period bands (high correlation) and drop down to zero over other bands (uncorrelated data). Let us assume here that the correlation is good as soon as $R^{2}$ is higher than e.g. 0.70: this permits to identify a so-called interval of sensitivity over $T_{-10}$. Then, the following comments may be formulated. Firstly, the curves - again - are very similar for both wave climates in a general way, even if the period range is not exactly the same for both since shorter waves are expected to propagate in the North Sea in comparison to the Atlantic Ocean [the figures for both locations have been placed side-by-side to help compare the results in each wave climate]. Secondly, some parameters exhibit higher $R^{2}$ values than others, namely $\varepsilon_{0}, \varepsilon_{1}$ and $B_{\mathrm{w}}$ (about 0.95 for IPS4 with $\varepsilon_{1}$, Fig. $9(\mathrm{~g} \& \mathrm{~h})$ ); on the contrary, parameters like $\varepsilon_{2}$ or $Q_{\mathrm{p}}$ do not 
seem particularly relevant from this point of view. Thirdly, the intervals of sensitivity for each parameter are quite similar at both locations: once again, the influence of the corresponding WEC's transfer function is significant. More precisely, the highest correlation values generally occur near the resonance peak. For example, IPS1 is highly resonant for waves of period 8-9s: in Figure 9(a\&b), the high values of $R^{2}$ range within [7s;10s]. Lastly, the intervals of sensitivity appear broader when the WEC's transfer function is broad. This is well observed when looking at configurations IPS3 and IPS4: for the first one - very narrow -, high values of coefficient $R^{2}$ only occur over reduced ranges of periods ( 2 s-wide intervals on average, Fig. 9(e\&f)) whereas for the second one - very broad -, the highest values are observed over very broad ranges (7s-wide intervals on average, Fig. 9(g\&h)).

Fig. 9 Determination coefficient $R^{2}$ of quadratic regressions (see Fig. 8) against energy period observed from long-term measurements in Figueira da Foz (left) and K13 (right) for several bandwidth parameters and for PTO configurations IPS1 to IPS4 (top to bottom); mean extracted power by the devices among each sea state sample (red spots, right axis)

As a conclusion, this study permits to understand that the bandwidth characteristic is relevant to complete the classical $H_{\mathrm{m} 0}-T_{-10}$ description of sea states, especially when the WEC's response is broad and tuned to the main frequency components of the incident wave field. In addition, this study shows that parameters such as $\varepsilon_{0}$ and $\varepsilon_{1}$ (among others, not all of the parameters introduced in Section 1 having been tested) are relevant to characterise the bandwidth of sea states in view of assessing the performance of such axi-symmetrical WECs.

\section{Sensitivity to spectral bandwidth of a weakly direction-sensitive three- dimensional WEC (SEAREV)}


The sensitivity to wave spectral bandwidth of the three-dimensional WEC SEAREV (see Babarit, 2005) is observed by means of a numerical simulator in the time domain developed in Ecole Centrale de Nantes. The device is a pitching body, thus not axisymmetrical and hence, subject to wave directionality. However, it is assumed and verified numerically that the model is very little sensitive to variations of directionality, at least within a $60^{\circ}$-wide sector (less than $3 \%$ on the mean extracted power in waves inducing the highest resonance).

\subsection{SEAREV time-domain simulation}

The physical WEC corresponds to hull DES1129 depicted in Figure 10(a\&b). The body is designed to oscillate in pitch as waves pass by. An inertial pendulum is located inside the hull: it freely moves around an axis parallel to the own hull's pitching axis, so that the relative pitch motion between both bodies $(\alpha)$ enables the extraction of energy from waves by means of a linear damper $\left(C_{\text {PTO }}=10^{7} \mathrm{~kg} \cdot \mathrm{m}^{2} \cdot \mathrm{s}^{-1}\right.$, Eq. (21)). Roll and yaw have been restricted with additional stiffness (roll: $K_{44}=10^{9} \mathrm{~N} \cdot \mathrm{m} \cdot \mathrm{rad}^{-1}$; yaw: $K_{66}=10^{8} \mathrm{~N} \cdot \mathrm{m} \cdot \mathrm{rad}^{-1}$ ) in order to favour pitching motions. The simulation code solves the integro-differential equation of Cummins (Cummins, 1962) with a $4^{\text {th }}$-order Runge-Kutta scheme. Radiation (added masses, impulse responses) and diffraction (wave excitation) efforts on the hull at rest - under deep water and small motions assumptions - are calculated in the time domain with the 3-D diffractionradiation code ACHIL3D (Clément, 1997). The model involves hydrodynamic non-linearities coming from the calculation of the instantaneous Froude-Krylov forces (fluid pressure forces exerted on the hull in undisturbed wave) at each time-step, which therefore justify the resort to time-domain modelling. 
Fig. 10DES1129 hull of the SEAREV WEC : 3-D view with inner pendulum (a) and side-view physical sketch (b) where $\theta$ and $\alpha$ respectively denote hull's pitch and pendulum's rotation angle (the body is approximately included in a $15 \mathrm{~m}$ side-length cube)

As the simulations in realistic sea states are somewhat time consuming, they are run several times over a short duration. Each run covers 500 s from rest position with the first $100 \mathrm{~s}$ being disregarded for they include a transient state from rest to random permanent regime. From a target directional spectrum $E(f, \theta)$, linear random wave fields are generated using the deterministic spectral amplitude method (see Miles and Funke, 1989), that is, selecting random phases for each frequency-direction component while wave amplitudes are computed according to

$$
A\left(f_{i}, \theta_{j}\right)=\sqrt{2 E\left(f_{i}, \theta_{j}\right) \Delta f_{i} \Delta \theta_{j}}
$$

With this method, running the simulator several times for a given target directional spectrum permits a faster convergence to the ensemble average value of extracted power related to the sea state, of which each simulation provides an estimate according to equation (22) applied to the angular velocity $d \alpha / d t$. In the following, the absolute performance results are given as distributions (mean and 80\% confidence interval).

\subsection{Wave spectral data}

The WEC's mean output power is estimated in nine weather sequences of sea states during the month of January 2007 near the coasts of Santa Barbara (California, U.S.A., see 
Fig. 11). This particular location $\left(34^{\circ} 16^{\prime} 21^{\prime \prime} \mathrm{N}, 120^{\circ} 41^{\prime} 55^{\prime \prime} \mathrm{W}\right)$ is referred to as buoy location 46063 by the American National Data Buoy Center (NDBC), and the local mean water depth is about $630 \mathrm{~m}$. The directional spectra are provided by the French Service Hydrographique et Océanographique de la Marine (SHOM) and were output from the global hindcast model WaveWatch III (WW3) using global ECMWF (European Centre for Mid-term Weather Forecasts) wind data. A preliminary study showed that WW3 and local field data (by NDBC directional buoy) were in very good agreement. The spectra are computed by WW3 every $3 \mathrm{~h}$.

Fig. 11 Californian coast near Los Angeles (USA) : location of NDBC buoy 46063 (Point Conception) [map drawn from NDBC website: www.ndbc.noaa.gov]

In the present sample, the sea states may exhibit more than one peak (i.e. wave system) for they are not necessarily unimodal. Figure 12 illustrates the time evolution of peak frequency for the whole spectrum as well as for the wave system (partition) that is likely to excite the WEC, as secondary or main peak over $[0.1 \mathrm{~Hz} ; 0.2 \mathrm{~Hz}]$. The nine identified sequences (1 to 9) underline the fact that the sea states mostly are bimodal: they mostly refer to wind-seas and a few ends of swell (6\&9). Indeed, wind-seas are characterised by high and rapidly decreasing peak frequencies against time, contrary to swells, whose peak frequency generally is low and slowly increasing. A finer analysis of the sea states - not reproduced here - reveal that both kinds of systems in this window arise from a restricted angular sector centred on $\sim 300^{\circ}$ (W-NW), which is used to set the WEC's orientation (fixed slack moorings) so that the bow is always facing the main waves.

Fig. 12 Peak frequency of both active wave system partition and whole spectrum at buoy station NDBC 46063 (January 2007, WW3 data) in nine weather sequences 


\subsection{Sensitivity of capture width to spectral bandwidth parameters}

Spectral bandwidth parameters $\varepsilon_{0}, \varepsilon_{2}, Q_{\mathrm{p}}, Q_{\mathrm{e}}, \Lambda$ and $\kappa$ are calculated for each simulated wave spectrum in the nine weather sequences. Capture width $\chi_{\mathrm{P}}$ is also computed according to equation (25), as

$$
\chi_{P}=\frac{\overline{P_{P T O}}}{P_{w}} \equiv \frac{\mathrm{E}\left[\hat{P}_{P T O}\right]}{P_{w}}
$$

where $\mathrm{E}[$.$] denotes ensemble average and the term with a hat denotes an estimation of the$ mean output power (in $\mathrm{kW}$ ) obtained from one single $(500 \mathrm{~s}-100 \mathrm{~s}=) 400 \mathrm{~s}-$ simulation of the WEC. For each sea state, about 30 of such simulations are run: the deterministic 3-D behaviour of the SEAREV is therefore calculated in about 30 random wave fields generated from the spectral components (Eq. (29)) and random phases. Figures 13(a-f) depict the scatter plot of $\chi_{\mathrm{P}}$ against each bandwidth parameter listed above.

Fig. 13 Scatter plots of SEAREV's capture width obtained in nine sequences (Fig. 12) against several bandwidth parameters (a to f) at station NDBC 46063 (January 2007)

Parameters $\varepsilon_{0}$ and $\varepsilon_{2}$ represent relative bandwidths: their use therefore only makes sense within sea state samples of the same mean wave period, as it is the case in Section 3 (Fig. 8). Yet here, sea states of different $T_{-10}$ are considered all together. Accordingly, it is not surprising to observe no clear correlation in Figure 13(a). Surprisingly however, Figure 13(b) exhibits some correlation between $\chi_{\mathrm{P}}$ and $\varepsilon_{2}$. The particular case of this narrowness parameter is more closely addressed in the following. 
Figures $13(\mathrm{c} \& \mathrm{~d})$ related to peakedness factors $Q_{\mathrm{p}}$ and $Q_{\mathrm{e}}$ are very similar - due to their own relative similarity (Eq. (4)\&(10)). Both exhibit a very neat $1 / x$ decreasing trend for capture width. The most "peaked" sea states (i.e. $Q_{\mathrm{p}}, Q_{\mathrm{e}}>2-3$ ) yield the weakest values of $\chi_{\mathrm{P}}$ ( $\sim 1$, i.e. as much power as the incident waves) whereas the broadest ones yield the highest performance levels ( $\chi_{\mathrm{P}}$ up to 7$)$. In such broad sea states, the peakedness asymptotically tends to its lowest value ( 1-2), for which the capture width varies a lot (from 2 to 7 ). However, let us remind that the calculation of such factors is theoretically valid in unimodal sea states only, which is not always the case here according to Figure 12.

Figures 13(e\&f) related to parameters $\Lambda$ and $\kappa$ inspire the same observation as previously: the weakest performance values correspond to the most narrow-banded sea states. A rough general correlation is found for $\Lambda$, which permits to estimate approximately the capture width from the knowledge of this parameter (in $\mathrm{Hz}$ ). For $\kappa$, a linear and homogeneous decreasing trend is observed as the successive wave height correlation increases $(\kappa>0.5)$. For lower correlation levels, the scatter of $\chi_{\mathrm{P}}$ may be quite important (1 to 7).

According to these observations, and looking back to the case of the relative bandwidth parameter $\varepsilon_{2}$ (Fig. 13(b)), the obtained scatter plot is particularly unexpected. Indeed, high values of $\varepsilon_{2}$ should correspond to very broad sea states, that is, to a high performance level. Here, the perfect inverse is observed, but still with a manifest correlation to $\chi_{\mathrm{P}}$. The computation of $\varepsilon_{2}$ must therefore be invoked to account for these results since no cut-off frequency has been applied in equation (6) on principle (integration up to the highest frequency of definition in $\mathrm{WW} 3,0.716 \mathrm{~Hz}$ ). This shows that parameter $\varepsilon_{2}$ remains very sensitive to the way it is calculated and requires particular care when computed. Therefore, it does not seem appropriate as standard bandwidth parameter for such purposes. 
So far, the response of WECs equipped with linear PTO devices has been considered exclusively. Such devices reproduce at the output the fluctuations of the power absorbed by the mechanical system since there is no inherent inertia in the model (Eq. (21)). The term inertia does not refer here to a property of the mechanical structure but is rather related to the short-term energy storage capacity induced by the electro-mechanical converter onboard. If one considers more realistic PTO devices such as hydraulic circuits with gas accumulators and hydraulic motors (Henderson, 2006; Falcão, 2007), air turbines (for oscillating water column systems, see Falcão, 2002), low-head water turbines (for overtopping systems, see Kofoed, 2002) etc. it is necessary to take this property into account. This allows for a smoothed or stabilized output power at the scale of one single WEC. Without inertia, a complex - a possibly expensive - power electronics assembling would be necessary to end up with a satisfactory power signal ready to be input into the grid. With inertia, this assembling is likely to be much reduced, and thus, much cheaper and easier to install. Accordingly, WEC developers have to find a reasonable compromise (performance/expenses/installation) on the level of inertia they wish inside their PTO device(s). It follows that the sensitivity of such systems to wave groupiness is of a particular concern. Here, two models are proposed, which aim at encompassing the most common PTO devices, namely inertial flywheels for turbines and short-term energy storage reservoirs for hydraulic installations.

\subsection{Inertial flywheel}

A simplified model of flywheel with adjustable inertia permits to reproduce the behaviour of air and water turbines. The instantaneous power absorbed by the mechanical 
system, denoted by $P_{\mathrm{a}}(t)\left(=P_{\mathrm{PTO}}(t)\right.$ for linear WECs in Sections $\left.2 \& 3\right)$, supplies energy to a flywheel that is linked to an electrical generator delivering the power $P_{\mathrm{e}}(t)$ (Fig. 14).

Fig. 14Simplified sketch of an inertial WEC equipped with flywheel and electrical generator

In the model, it is assumed that the output electrical power is related to the flywheel's instantaneous rotational speed $\Omega(t)(\mathrm{rad} / \mathrm{s})$ by the relation

$P_{e}(t)=K \Omega^{2}(t)$

where $K\left(\mathrm{~kg} \cdot \mathrm{m}^{2} / \mathrm{s}\right)$ is a constant that can be freely adjusted (control law). By neglecting energy losses by friction on the rotor, the dynamic equation of the PTO is (Falcão, 2002)

$P_{a}(t)-P_{e}(t)=I \Omega(t) \cdot \frac{d \Omega(t)}{d t}$

where $I\left(\mathrm{~kg} \cdot \mathrm{m}^{2}\right)$ denotes the flywheel's inertia against the rotation axis. Combining both equations (31)\&(32) leads to the following dynamic equation

$$
\begin{aligned}
P_{a}(t) & =P_{e}(t)+\frac{I}{2 K} \cdot \frac{d P_{e}(t)}{d t} \\
& =P_{e}(t)+\mu \cdot \frac{d P_{e}(t)}{d t}
\end{aligned}
$$

The inertia of the PTO can be characterised here as the time constant $\mu=I / 2 K(\mathrm{~s})$. Indeed, equation (33) is similar to that of an $\mathrm{RC}$ electrical circuit with resistor $R(\equiv 1 / 2 K)$ and capacitor $C(\equiv I)$, for which the product $\eta=R C$ represents the circuit's time constant, i.e. the time 
required to reach $\sim 63 \%$ of the permanent regime voltage. For a given control law $(K)$, the higher the flywheel's inertia $(I)$, the higher the level of energy storage at short term $(\mu)$.

With such a model, it is immediately apparent that the (long term) expected mean power converted by the whole WEC is the same as for the linear model - denoted here by $P_{\mathrm{a}, \mathrm{m}}$ in this section (Eq. (24)) - , since no energy loss is included. Thus, at the scale of a sea state, this WEC has a similar sensitivity to spectral bandwidth as any of the linear models considered so far (Sections 3\&4). Now, the instantaneous response will differ somewhat. Indeed, the PTO system constitutes a low-pass filter, which therefore reduces the highfrequency fluctuations of the absorbed power. The resulting electrical power signal $P_{\mathrm{e}}(t)$ appears then smoother than the instantaneous input power $P_{\mathrm{a}}(t)$ as well as slightly delayed in time. Figures $15(\mathrm{a} \& \mathrm{~b})$ give an example of this signal for three levels of inertia, as $\mu=5 \mathrm{~s}$ (low inertia), $\mu=25 \mathrm{~s}$ (mean inertia) and $\mu=100 \mathrm{~s}$ (high inertia), and respectively denoted by $P_{\mathrm{e} 1}(t)$, $P_{\mathrm{e} 2}(t)$ and $P_{\mathrm{e} 3}(t)$. The input absorbed power $P_{\mathrm{a}}(t)$ comes from the stochastic simulation over $3600 \mathrm{~s}$ of the axi-symmetrical heaving buoy introduced in Section 2 in a unimodal $\left(H_{\mathrm{m} 0}=2 \mathrm{~m}\right.$, $T_{\mathrm{p}}=8 \mathrm{~s}$ ) and bimodal (swell: $H_{\mathrm{m} 0}=1.41 \mathrm{~m}, T_{\mathrm{p}}=11 \mathrm{~s}$; wind-sea: $H_{\mathrm{m} 0}=1.41 \mathrm{~m}, T_{\mathrm{p}}=5 \mathrm{~s}$ ) sea state respectively, using random Fourier coefficients (see Tucker et al., 1984; Miles and Funke, 1989). Both target spectra are plotted in Figure 16 , and have similar $H_{\mathrm{m} 0}(2 \mathrm{~m})$ and $T_{-10}(\sim 7 \mathrm{~s})$. In these excerpts, the wave signal together with the instantaneous energy histories $\operatorname{SIWEH}(t)$ and $I E(t)$ as well as the spectral densities of each electrical power signal are also plotted.

Fig. 15 Simulation of WEC equipped with flywheel in unimodal (a) and bimodal (b) target sea states: wave record and related wave energy histories SIWEH and IE (top), instantaneous power extracted by the WEC $(\mu=0,5,25$ and 100s, middle), related power variance spectra (bottom) 
Both simulations emphasize the smoothing effect realized by the PTO device with respect to the non-filtered input power signal $P_{\mathrm{a}}(t)$. As inertia $\mu$ increases, the level of smoothing is more and more important. Thus, $P_{\mathrm{e} 3}(t)$ (high inertia) is almost constant around the expected mean power value $(\sim 28 \mathrm{~kW}$ and $\sim 20 \mathrm{~kW}$ in both sea states respectively). On the contrary, $P_{\mathrm{e} 1}(t)$ (low inertia) still varies a lot and exhibits high peaks $(>100 \mathrm{~kW}$ here in Fig. 15a). According to these plots, the sensitivity of the power signals to wave groups at short term - identified by $\operatorname{SIWEH}(t)$ and $I E(t)$ - is manifest and decreases with inertia $\mu$. In order to validate this observation, the inter-correlation function of centred and normalised signals $P_{\mathrm{e} 1}(t), P_{\mathrm{e} 2}(t)$ and $P_{\mathrm{e} 3}(t)$ with the centred and normalised instantaneous wave energy signals $\operatorname{SIWEH}(t)$ and $I E(t)$ is plotted in Figures $17(\mathrm{a}-\mathrm{d})$. These curves are respectively obtained for the same target unimodal and bimodal sea states as previously (Fig. 16). For both wave energy signals and both sea states, it is observed that the inter-correlation peak becomes higher and higher as inertia decreases: hence, the best correlation is found for the low inertia configuration $\mu=5 \mathrm{~s}$. Moreover, the delay induced by each level of inertia is clearly emphasized and increases with inertia, as approximately $5 \mathrm{~s}, 8 \mathrm{~s}$ and $20 \mathrm{~s}$ for $P_{\mathrm{e} 1}(t), P_{\mathrm{e} 2}(t)$ and $P_{\mathrm{e} 3}(t)$ respectively in the unimodal sea state. Similar delays are obtained in the bimodal case. This means that an optimal inertia may be setup for the flywheel in such sea states by adjusting the control law $(K)$ according to the desired degree of sensitivity to wave groups. This short-term sensitivity, however, is not supposed to modify the mean converted power by the WEC in the whole sea state (i.e. in 1-3h of simulation), as already mentioned previously. 
Fig. 17Intercorrelation function of (normalized and centred) instantaneous power signals output from the flywheel with instantaneous wave energy histories SIWEH (a\&c) and IE (b\&d) in unimodal (top) and bimodal (bottom) sea states (Fig. 16)

\subsection{Short-term energy storage with nominal output power}

The second way of simulating an inertial PTO device is by considering a simple shortterm potential energy reservoir supplied by the same instantaneous power $P_{\mathrm{a}}(t)$ as previously (instantaneous power absorbed by the linear heaving buoy) and connected to a hydraulic motor characterised by the nominal output power $P_{\text {nom }}(\mathrm{kW})$. The instantaneous power output by the whole WEC is denoted by $P_{\mathrm{s}}(t)$ : either it is equal to $P_{\text {nom, }}$, when energy is discharged by the reservoir through the motor, or it is zero, when the energy stored inside the reservoir $\left(E_{\text {capa }}(t)\right)$ is not sufficient to actuate the motor. A straightforward algorithm is built to simulate the whole WEC (see Saulnier, 2009), which is illustrated in Figure 18. No maximal limit is imposed on the reservoir's capacity. A 1200s-simulation of the WEC in the same target unimodal sea state as previously $\left(H_{\mathrm{m} 0}=2 \mathrm{~m}, T_{\mathrm{p}}=8 \mathrm{~s}\right)$ is shown in Figure 19, where $P_{\text {nom }}$ is set to the expectation $P_{\mathrm{a}, \mathrm{m}}$ of $P_{\mathrm{a}}(t)$ in the sea state $(\sim 28 \mathrm{~kW}$, Eq. (24)) : as expected, the output power is intermittent, depending on the available energy inside the reservoir.

Fig. 18Simplified sketch of an inertial WEC equipped with short-term energy reservoir and hydraulic motor with nominal power

Fig. 19Simulation of WEC equipped with short-term energy reservoir in unimodal target sea state (Fig. 16): wave record and buoy's heave motions (top), instantaneous power extracted 
by the WEC with and without reservoir (middle), and instantaneous stored energy inside the reservoir (bottom)

In order to characterise the performance of the whole system in a sea state, the quality factor (or nominal operating rate, \%) is defined and calculated as

$$
\Phi_{q, P \text { nom }}(T)=\frac{\sum \tau\left(P_{s}=P_{\text {nom }}\right)}{T}[\%]
$$

as a function of the simulation length $T(\mathrm{~s})$. This factor also depends on the nominal power of

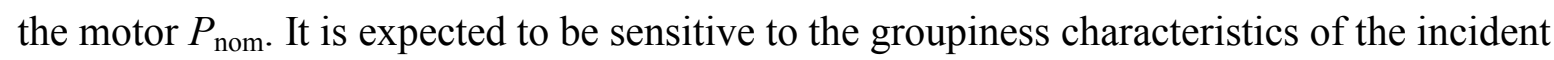
wave field and, therefore, may constitute a relevant wave group parameter related to wave energy extraction. For the sake of simplicity, $P_{\text {nom }}$ by default is taken equal to $P_{\mathrm{a}, \mathrm{m}}-$ as in Figure 19 - and the related quality factor is more conveniently denoted by $\Phi_{\mathrm{q}}(T)$. Figure 20 depicts the expectation of $\Phi_{\mathrm{q}}$ along with the estimates' dispersion against $T$ from 300simulations samples in the unimodal sea state obtained with two wave simulation methods (random Fourier coefficients method "a", and deterministic spectral amplitude method "b"). This figure shows that: 1 / whatever the method, the expectation of $\Phi_{\mathrm{q}}$ increases with $T$, and $2 /$ the factor does not seem to reach a convergent asymptotical value $\Phi_{\mathrm{q}}(\infty)$. In the following, wave simulation method "a" is adopted for the estimation of $\Phi_{\mathrm{q}}(T)$.

Fig. 20 Expectation (left axis) and standard deviation (right axis) of quality factor $\Phi_{q}$ against simulation length by simulating waves with random Fourier coefficients (method a) and random phases only (method b) 
Let us now observe the sensitivity of the quality factor to the bandwidth of sea states with the same $H_{\mathrm{m} 0}$ and $T_{-10}$. Three PTO configurations are envisioned: $(a) P_{\mathrm{nom}}=P_{\mathrm{a}, \mathrm{m}}$ (default), (b) $P_{\mathrm{nom}}=P_{\mathrm{a}, \mathrm{m}} / 5$ (overloaded, saturation) and $(c) P_{\mathrm{nom}}=P_{\mathrm{a}, \mathrm{m}} * 5$ (underloaded). Using generalized Bretschneider shapes as target spectra (see Eq. (28) and Fig. 22), Figures 21(a-c) depict the expected value of factor $\Phi_{\mathrm{q}, \text { Pnom }}$ estimated for various simulation lengths $\left(600,1200,1800\right.$ and 3600s) against factor $Q_{\mathrm{p}}$, which governs the peakedness of the spectra. The corresponding values of bandwidth parameters $\varepsilon_{2}, \Lambda(\mathrm{Hz})$ and $\varepsilon_{\dot{z}, 2}$ (heave velocity motions bandwidth) are added to the plots corresponding to $Q_{\mathrm{p}}$ values ranging from 1 to 7 .

Fig. 21 Expectation of quality factor against peakedness factor $Q_{p}$ (unimodal sea states) for several simulation lengths (600 to 3600s) and three operating situations: nominal case $P_{\text {nom }}=$ $P_{\mathrm{a}, \mathrm{m}}(a)$, saturation case $P_{n o m}=P_{\mathrm{a}, \mathrm{m}} / 5$ (b) and underload case $P_{\text {nom }}=P_{\mathrm{a}, \mathrm{m}} * 5$ (c); spectral bandwidth parameters (wave and motions)

In the default configuration case $(a)$, a visible sensitivity of $\Phi_{\mathrm{q}}$ to $Q_{\mathrm{p}}$ is observed: the expected value of the factor decreases as peakedness increases (of about 4-6\%). Thus, the broader the wave spectrum, the better the motor's operating rate. Let us remember, however, that the mean power absorbed by the buoy in each sea state is different, as approximately $17 \mathrm{~kW}$ for $Q_{\mathrm{p}}=1$ and $40 \mathrm{~kW}$ for $Q_{\mathrm{p}}=7$. In the overloaded case $(b)$, this sensitivity is not observed anymore since very similar values of mean quality factor are found whatever the simulation length - and very close to $100 \%$, as expected. In the underloaded case $(c)$, the sensitivity to $Q_{\mathrm{p}}$ is not observed either: here again, $\Phi_{\mathrm{q}}$ does not vary a lot against peakedness, while its value is now very low $(<20 \%)$. As a conclusion, in nominal conditions (i.e. when the device is tuned to the main wave components, see Fig. 22, and $P_{\text {nom }} \sim P_{\mathrm{a}, \mathrm{m}}$ ), the motor's operating rate is related to the spectral bandwidth: it is found higher in broad-banded sea states. In critical conditions, that is, when the system saturates (i.e. $\Phi_{\mathrm{q}} \sim 100 \%$ while the 
stored energy diverges) or when the sea state is too weak to fill up the reservoir with potential energy (i.e. low $\Phi_{\mathrm{q}}$ ), the bandwidth of waves does not matter anymore.

Fig. 22 Target unimodal variance spectral densities with modulable peakedness factor $\left(Q_{p}=\right.$ 1 to 7) used in Fig. 21; dimensionless WEC's power transfer function

\section{Conclusions}

This work addressed the question of the sensitivity of WECs to wave groupiness and spectral bandwidth of sea states in addition to the common wave parameters $H_{\mathrm{s}}$ and $T_{\mathrm{e}}$ (respectively denoted by $H_{\mathrm{m} 0}$ and $T_{-10}$ in this paper), in particular when the devices are little influenced by wave directionality (point absorbers). To this end, linear stochastic modelling and non-linear time-domain simulations have been carried out involving linear PTO devices (linear damping). The performance results have led to the following conclusions.

Firstly, for fixed $H_{\mathrm{m} 0}$ and $T_{-10}$ in any wave climate, the variability of the performance symbolized by a capture width parameter $\left(\chi_{\mathrm{P}}\right.$, in $\left.\mathrm{m}\right)$ - can be very important, especially in sea states whose energy period lies within the response band of the WEC (PTF) due to the shape variability of wave spectra in nature. Secondly, the spectral bandwidth of waves - which is related to the wave groupiness phenomenon through the spectral narrowness - is found to adequately completes the $\left(H_{\mathrm{m} 0}, T_{-10}\right)$ sea state description for characterising the converter's performance, in particular when the mean period of the incoming waves is close to the converter's resonance. Thirdly, the sensitivity of a WEC to spectral bandwidth is found to be more pronounced when its response band is broad (§3.3.2, Fig. 9). This, together with the last point, implies that, if the WECs are designed in such a way their response band is broad and may be automatically tuned to the main wave periods of each experienced sea state, the 
spectral bandwidth will constitute the missing key parameter which provides a comprehensive description of the resource as regards the wave energy conversion operated by the device. According to the models, some spectral bandwidth parameters have shown to behave satisfactorily for this purpose. In the case of the linear IPS model, relative bandwidth parameters such as $\varepsilon_{0}$ and $\varepsilon_{1}$ appeared adequate, particularly in sea states with same energy period $T_{-10}$. From the simulations of the SEAREV 3-D model, parameters and factors like $\Lambda, \kappa$ and $Q_{\mathrm{p}}$ or $Q_{\mathrm{e}}$ were found to be quite correlated with the performance, especially when the wave field is narrow-banded.

The consideration of realistic non-linear PTO devices inducing inertia within the energy conversion chain also has been carried out. Two different systems were envisioned and simply modelled in the time domain as connected to the output of a linear 1-DOF axisymmetrical buoy. The first one of them permits to reproduce the flywheel effect with controllable wheel inertia, which smoothes the power output from the linear buoy. By construction, the induced delay of the output power signal with respect to the raw one - that is, that obtained by linear conversion of the buoy' heave motions - may only modify the response of the device at short term: the sensitivity to wave groups can be easily identified thanks to instantaneous wave energy signals like $S I W E H$ or $I E$ (from EMD-HHT) as soon as the wheel's inertia is not too important. Indeed, it has been observed that the inter-correlation of the power output with the incident waves decreases with inertia. At long term, the mean output power is not expected to be influenced by the flywheel effect, out of technical issues linked to inner energy dissipation, working limits, stall effect, etc. which are too much system-specific to be incorporated in this study. The second PTO device model reproduced the behaviour of a hydraulic system composed of a reservoir of potential energy and a hydraulic motor with nominal power. It has been shown that the motor's working rate (quality factor) related to a given simulation length increases with the bandwidth of the sea state when the WEC is tuned to the main waves and when the nominal power is close to the mean power 
absorbed by the mechanical system without energy storage. In any other case, wave groupiness and spectral bandwidth are not, a priori, influential characteristics.

This study has therefore highlighted the capital role played by wave groupiness and spectral bandwidth in the behaviour of WECs as well as any offshore structure, for these characteristics - still hardly regarded in offshore engineering in general - may particularly influence their performance at both short and long term. The introduction of a new parameter in resource assessment would permit to refine the characterisation of sea states in view of predicting better the performance of WECs, at least those that are little sensitive to wave directionality.

\section{Acknowledgements}

This work has been partly funded by the E. C. WAVETRAIN Research Training Network Towards Competitive Ocean Energy, contract n MRTN-CT-2004-50166 (Marie Curie Actions) and the French Région Pays-de-la-Loire. Co-author António F. de O. Falcão acknowledges the support of I.D.M.E.C.. The authors also thank the Dutch Rijkswaterstaat and the French SHOM for the free use of North Sea Wavec Buoy and Californian WaveWatch III wave data respectively.

\section{References}

Ahran, M., Ezraty, R., 1978. Statistical relations between successive wave heights. Oceanologica Acta, 1, 151-158. 
Babarit, A., 2005. Optimisation hydrodynamique et contrôle optimal d'un récupérateur d'énergie des vagues. Ph.D. Thesis, Ecole Centrale de Nantes, France.

Battjes, J. A., Vledder (van), G. P., 1984. Verification of Kimura's theory for wave group statistics. In: Proc. of Int. Conf. on Coast. Eng. '84, pp. 642-648.

Blackman, R. B., Tukey, J. M., 1959. The measurement of power spectra. Dover Publications Inc., New-York, U.S.A.

Clément, A. H., 1997. Hydrodynamique instationnaire linéarisée : mise en œuvre d'une méthode de singularités utilisant un modèle différentiel de la fonction de Green. Tech. Rep. LHN-9703, Laboratoire de Mécanique des Fluides, Ecole Centrale de Nantes, France.

Cummins, W. E., 1962. The impulse response function and ship motions. Schiffstechnik, 9 (1661), 101-109.

Dong, G.-H., Ma Y.-X., Ma, X.-Z., 2008. Cross-shore variations of wave groupiness by wavelet transform. Ocean Eng., 35, 676-684.

Ewing, J. A., 1973. Mean length of runs of high waves. J. of Geophys. Res., 78, 1933-1936.

Falcão, A. F. de O., 2002. Control of an oscillating-water-column wave power plant for maximum energy production. Appl. Ocean Res., 24, 73-82.

Falcão, A. F. de O., 2007. Modelling and control of oscillating-body wave energy converters with hydraulic power take-off and gas accumulator. Ocean Eng., 34, 2021-2032.

Falnes, J., 2002. Ocean waves and oscillating systems: linear interactions including waveenergy extraction. Cambridge University Press, Cambridge, United Kingdom.

Funke, E. R., Mansard, E. P. D., 1980. On the synthesis of realistic sea states. In: Proc. of Int. Conf. on Coast. Eng. '80, pp. 2974-2991.

Goda, Y., 1976. On wave groups. In: Proc. of $1^{\text {st }}$ Behav. of Offshore Struct. Conf. '76, The Norwegian Institute of Technology. 
Guedes Soares, C., 1984. Representation of double-peaked sea wave spectra. Ocean Eng., 11, 185-207.

Hanson, J. L., Phillips, O. M., 2001. Automated analysis of ocean surface directional wave spectra. J. of Atmos. and Ocean Tech., 18, 277-293.

Hasselmann, K., Barnett, T. P., Bouws, E., Carlson, H., Cartwright, D. E., Enke, K., Ewing, J. A., Gienapp, H., Hasselmann, D. E., Kruseman, P., Meerburg, A., Müller, P., Olbers, D. J., Richter, K., Sell, W., Walden, H., 1973. Measurements of wind-wave growth and swell decay during the joint North Sea wave project (JONSWAP). Deutsche Hydr. Zeit. $\left(8^{\circ}\right)$, A12, 1-95.

Henderson, R., 2006. Design, simulation, and testing of a novel hydraulic power take-off system for the Pelamis wave energy converter. Renew. Energy, 31, 271-283.

Hogben, N., Lumb, F. E., 1967. Ocean waves - Statistics. H.M.S.O., London, United Kingdom.

Huang, N. E., Shen, Z., Long. S. R., Wu. M. C., Shih, H. H., Zheng, Q., Yen, N.-C., Tung, C. C., Liu, H. H., 1998. The empirical mode decomposition and the Hilbert spectrum for nonlinear and non-stationary time series analysis. In: Proc. R. Soc. Lond., A, 454, pp. 903-995.

Kerbiriou, M.A., 2007. Energie des vagues: méthodologie d'estimation du potentiel d'énergie récupérable par un dispositif d'extraction d'énergie des vagues au regard du potentiel naturel du site. Final post-doct. Rep. Ifremer ERT/HO: R05 HO 07, conv. ADEME n ${ }^{\circ}$ 0505 C0156, IFREMER Brest, France.

Kerbiriou, M.A., Prevosto, M., Maisondieu, C., 2007. Influence of an improved sea-state description on a wave energy converter production. In: Proc. of $26^{\text {th }}$ Int. Conf. on Offshore Mech. and Arct. Eng., San Diego, California, USA, June 07.

Kimura, A., 1980. Statistical properties of random wave groups. In: Proc. of Int. Conf. on Coast. Eng.'80, Sydney, Australia, pp. 2955-2973. 
Kofoed, J. P., 2002. Wave overtopping of marine structures - utilization of wave energy. PhD Thesis Rep., Aalborg University, Denmark, 189pp.

List, J. H., 1991. Wave groupiness in the nearshore. Coast. Eng., 5, 475-496.

Longuet-Higgins, M. S., 1957. The statistical analysis of a random moving surface. Phil.

Trans. R. Soc. Lond., A, 249, 321-387.

Longuet-Higgins, M. S., 1984. Statistical properties of wave groups in a random sea state.

Phil. Trans. R. Soc. Lond., A, 312, 219-250.

Medina, J. R., Hudspeth, R. T., 1987. Sea states defined by wave height/period functions. In: Proc. of Int. Assoc. for Hydraul. Res. Congress, Seminar on wave analysis and generation in laboratory basins, Lausanne, Switzerland, pp. 249-259.

Medina, J. R., Hudspeth, R. T., 1990. A review of the analyses of ocean wave groups. Coast. Eng., 14, 515-542.

Miles, M. D., Funke, E. R., 1989. A comparison of methods for synthesis of directional seas. J. of Offshore Mech. and Arct. Eng., 111, 43-48.

Mollison, D. 1985. Wave climate and the wave power resource. In: Evans, D.V., and Falcão, A.F.O. (eds.), Hydrodyamics of Ocean-Wave Energy Utilization, Proc. of IUTAM Symp., Lisbon, Portugal, pp. 133-156.

Nolte, K. G., Hsu, F. H., 1972. Statistics of ocean wave groups. In: Proc. of $4^{\text {th }}$ Offshore Tech. Conf., paper No. 1688, 2, pp. 637-644.

Ochi, M. K., Hubble, E. N., 1976. On six-parameters wave spectra. In: Proc. of Int. Conf. on Coast. Eng.'76, pp. 301-328.

Pierson, W. J., Moskowitz, L., 1964. A proposed spectral form for fully developed wind sea based on the similarity theory of S. A. Kitaigorodskii. J. of Geophys. Res., 69, 51815190. 
Pontes, M. T., Barstow, S., Bertotti, L., Cavaleri, L., Oliveira-Pires, H., 1997. Use of numerical wind-wave models for assessment of the offshore wave energy resource. J. of Offshore Mech. and Arct. Eng., 119, 184-190.

Pontes, M. T., 1998. Assessing the European wave energy resource. J. of Offshore Mech. and Arct. Eng., 120, 226-231.

Portilla, J., Ocampo-Torres, F. J., Monbaliu, J., 2009. Spectral partitioning and identification of wind sea and swell. J. of Atmos. and Ocean Tech., 26, 107-122.

Prevosto, M., 1988. Groupage de vagues et excitation basse fréquence - Etude ARAE. Tech. rep., IFREMER Brest, France.

Rice, S. O., 1944. The mathematical analysis of random noise. Bell Syst. Tech. Jour., 23, 282332.

Rice, S. O., 1945. The mathematical analysis of random noise. Bell Syst. Tech. Jour., 24, 46156.

Rye, H., 1974. Wave groups formation among storm waves. In: Proc. of Int. Conf. on Coast. Eng.'74, pp. 164-183.

Sawnhey, M. D., 1963. A study of ocean wave amplitudes in terms of the theory of runs and a Markov chain process. Tech. Rep., Dept. of Meteorology and Oceanography, NewYork University, U.S.A.

Saulnier, J.-B., 2009. Refined wave climatology as a contribution to the design of wave energy conversion systems. Ph.D. Thesis Rep., Ecole Centrale de Nantes, France, and Instituto Superior Técnico, Lisbon, Portugal, 258pp.

Smith, G. H., Venugopal, V., Wolfram, J., 2006. Wave period group statistics for real sea waves and wave energy extraction. Proc. of Int. Mech. Eng., 220, part M: J. of Eng. for the Marit. Environ., 99-115.

Torsethaugen, K. Haver, S, 2004. Simplified double peak spectral model for ocean waves. In: Proc. of Int. Symp. on Offshore. and Polar Eng..'04, Toulon, France. 
Tucker, M. J., Challenor, P. G., Carter, D. J. T., 1984. Numerical simulation of a random sea: a common error and its effect upon wave group statistics. Appl. Ocean Res., 6, 118122.

Woolf, D., 2002. Sensitivity of power output to wave spectral distribution. Tech. Rep., Seapower Ltd.

URLs (last visit on the $22^{\text {nd }}$ of March 2010)

ANEMOC: $\quad$ http://anemoc.cetmef.developpement-durable.gouv.fr/

ECMWF: $\quad$ http://www.ecmwf.int/

NOAA/NDBC: $\quad$ http://www.ndbc.noaa.gov/

Rijkswaterstaat: $\quad$ http://www.rijkswaterstaat.nl/

SHOM: $\quad$ http://www.shom.fr/

WERATLAS:

http://www.ineti.pt/download.aspx?id=23D554A7272EB9BC553C12D3D78B845D 


\begin{tabular}{|c|c|c|}
\hline Characteristics & $F F$ & K13 \\
\hline Location & $40^{\circ} 13^{\prime} 33^{\prime \prime} \mathrm{N}, 09^{\circ} 06^{\prime} 00^{\prime \prime} \mathrm{W}$ & $53^{\circ} 12^{\prime} 17^{\prime \prime} \mathrm{N}, 03^{\circ} 03^{\prime} 10^{\prime \prime} \mathrm{E}$ \\
\hline Water depth & $\sim 90 \mathrm{~m}$ & $\sim 30 \mathrm{~m}$ \\
\hline Measuring device(s) & Dir. and non-dir. Waveriders & Dir. Wavec \\
\hline Time coverage & $1981-1994$ & 1993-2002 \\
\hline Recording rate & $3 \mathrm{~h}$ & $3 \mathrm{~h}$ \\
\hline Number of collected spectra ${ }^{a}$ & $26500 \mathrm{sp}$. & $23300 \mathrm{sp}$. \\
\hline
\end{tabular}

${ }^{a}$ after removal of extra- and erroneous recordings

Table 1: In situ wave data: characteristics of buoy measurement campaigns carried out in Portugal (Figueira da Foz) and in the North Sea (K13 station) 


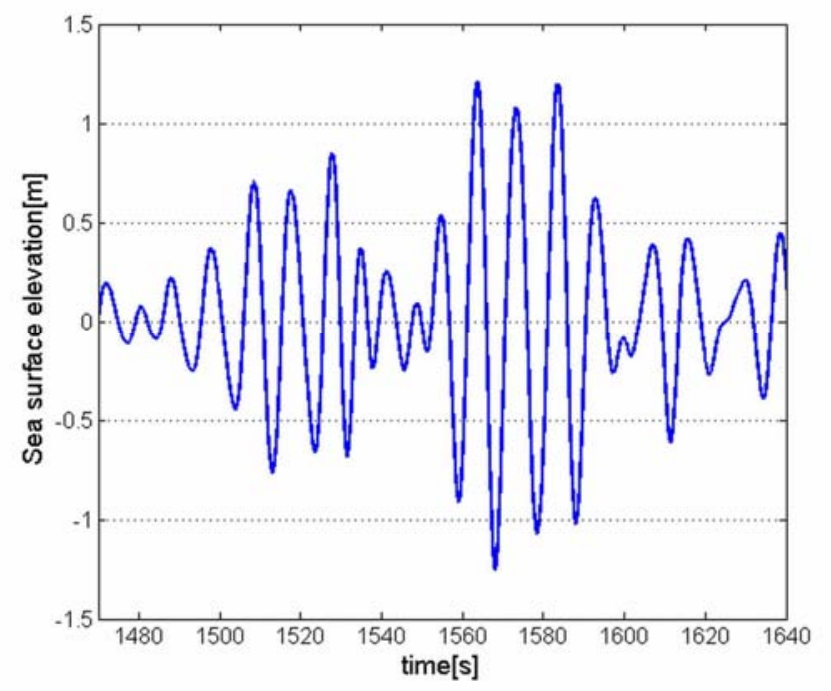

Fig 1: Sequence of two wave groups whose heights are superior to $1 \mathrm{~m}$ in a wave record 

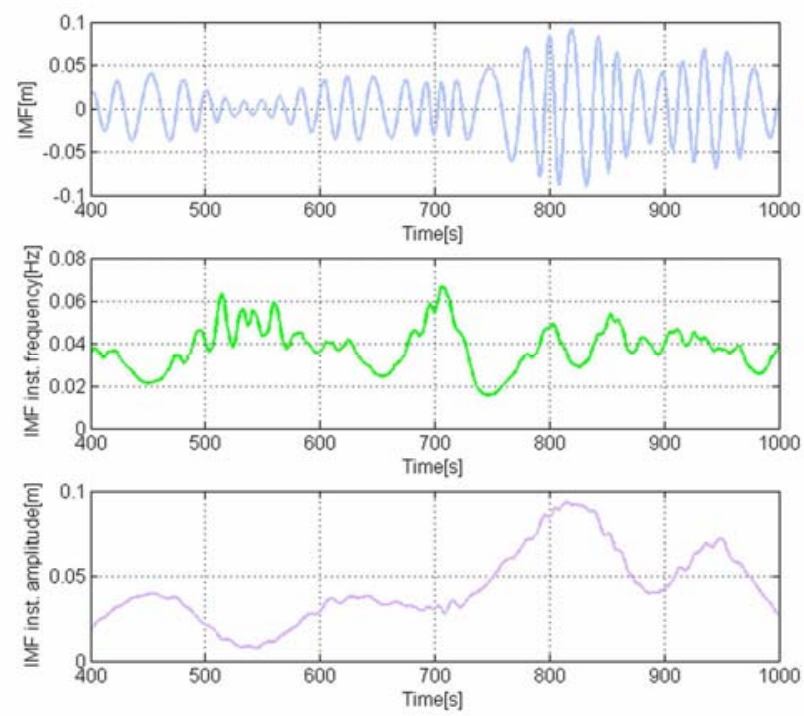

Fig. 2: Extract of IMF (top) derived by EMD-HHT from a wave signal, with related instantaneous frequency (middle) and amplitude (bottom) 
Click here to download Figure: Fig_03_pdf.pdf

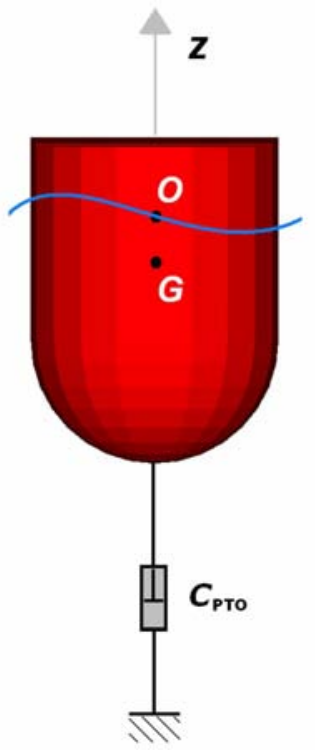

Fig. 3: Physical sketch of the one degree-of-freedom WEC oscillating in heave 


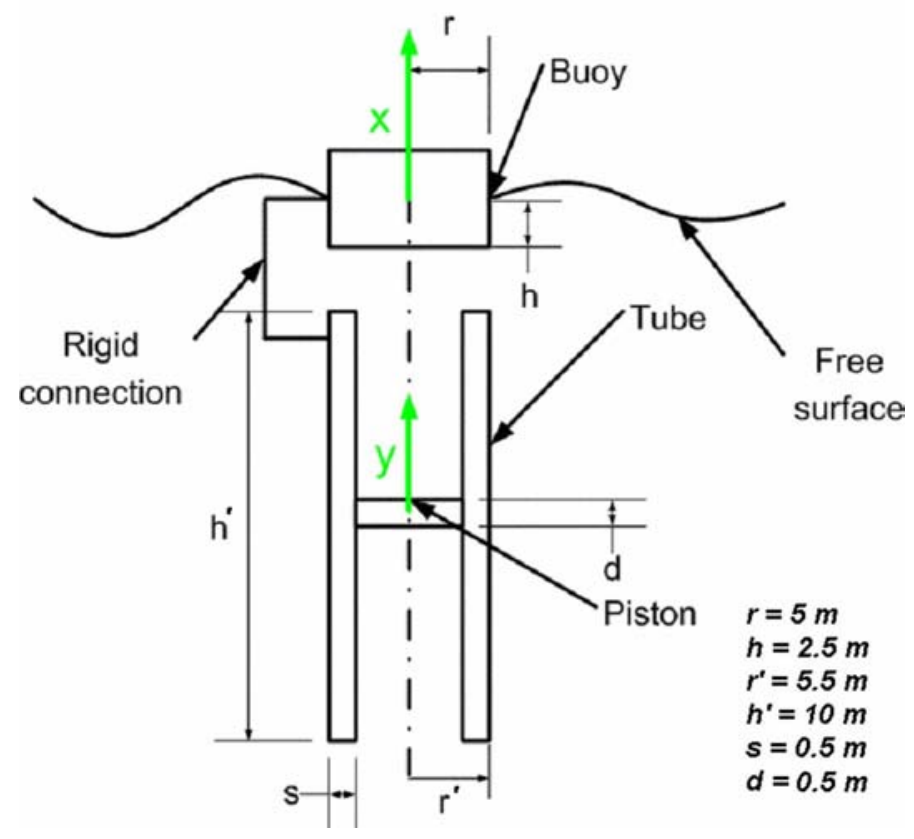

Fig. 4: Sketch of the two degrees-of-freedom axisymmetrical IPS WEC 


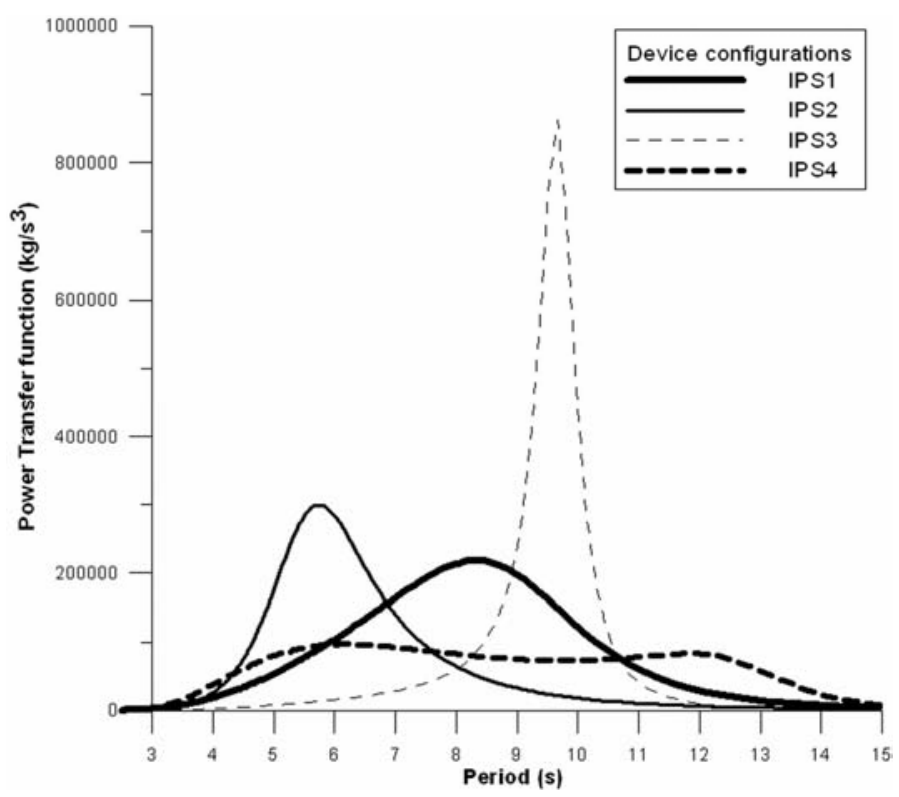

Fig. 5: Power transfer functions of the four linear PTO configurations against wave period 


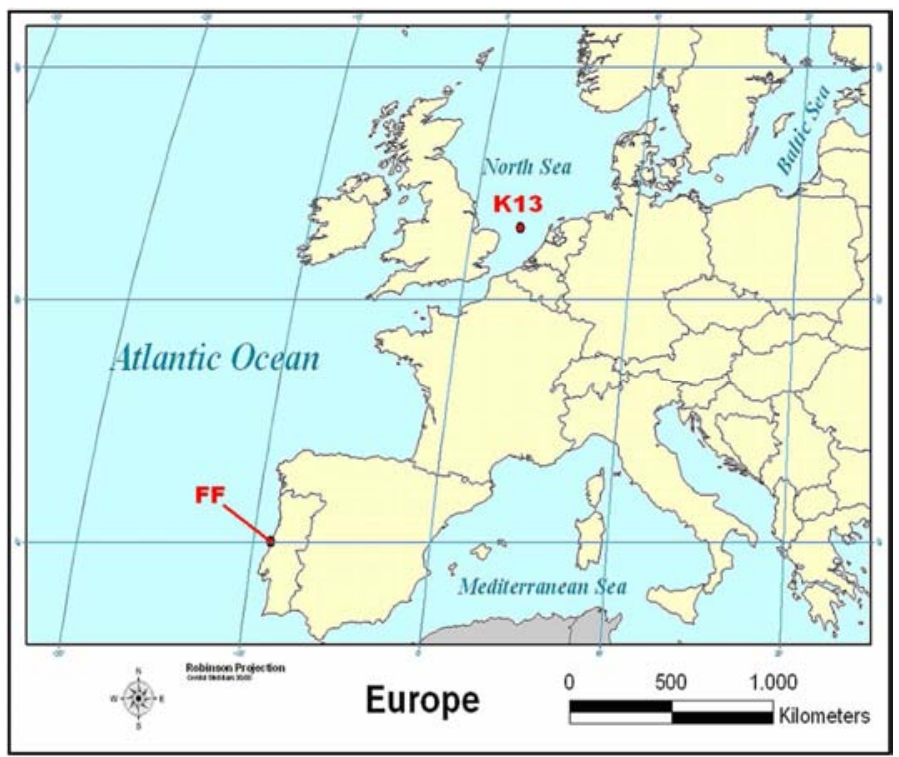

Fig. 6: Location of the buoys deployed off Figueira da Foz (Portugal) and near the K13 platform (North Sea) 

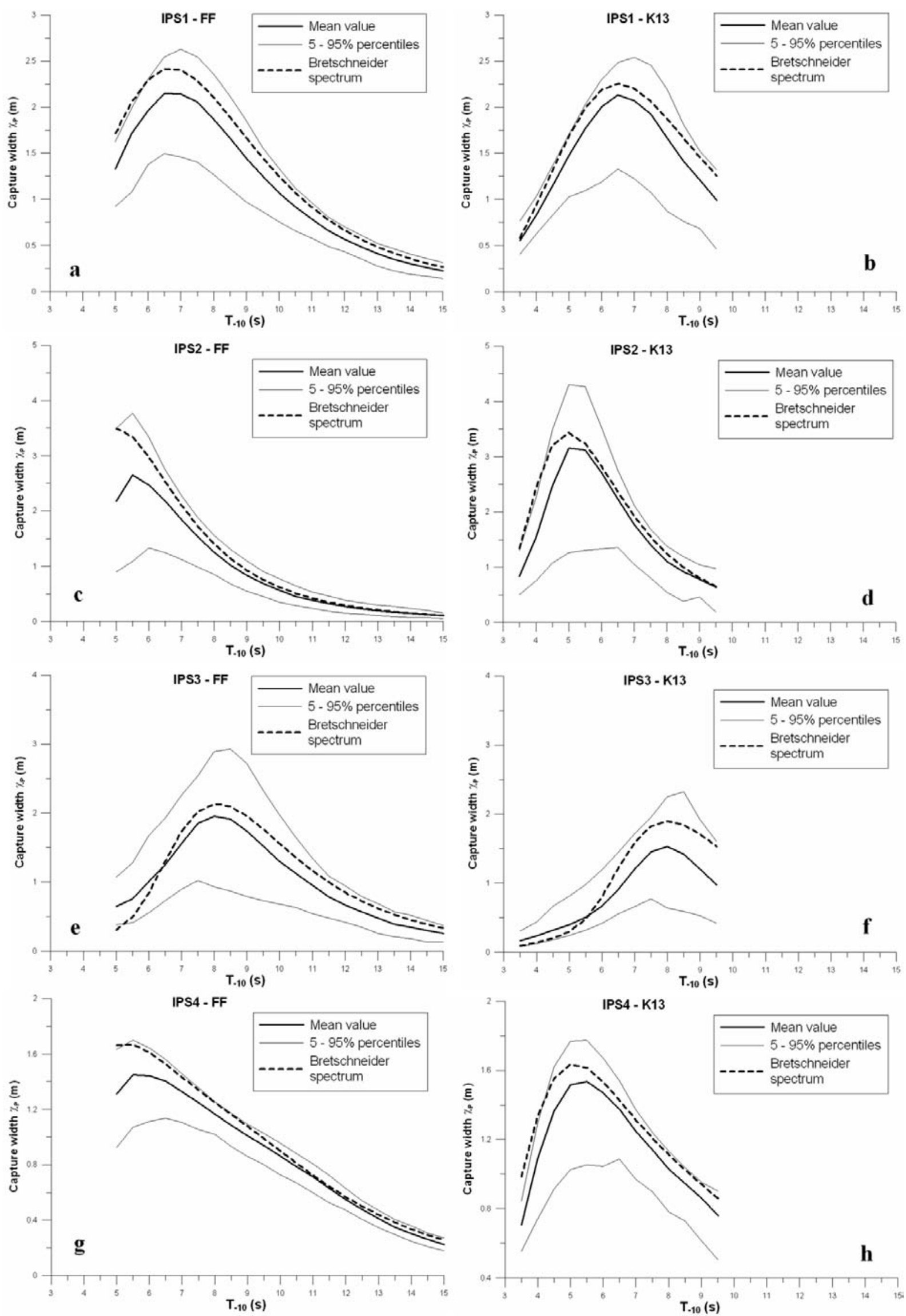

Fig. 7: Distribution of capture width against mean energy period obtained from long-term wave measurements in Figueira da Foz (left) and K13 (right) for PTO configurations IPS1 to IPS4 (top to bottom); capture width obtained with Bretschneider sea states (dotted line) 
Click here to download Figure: Fig_08_pdf.pdf

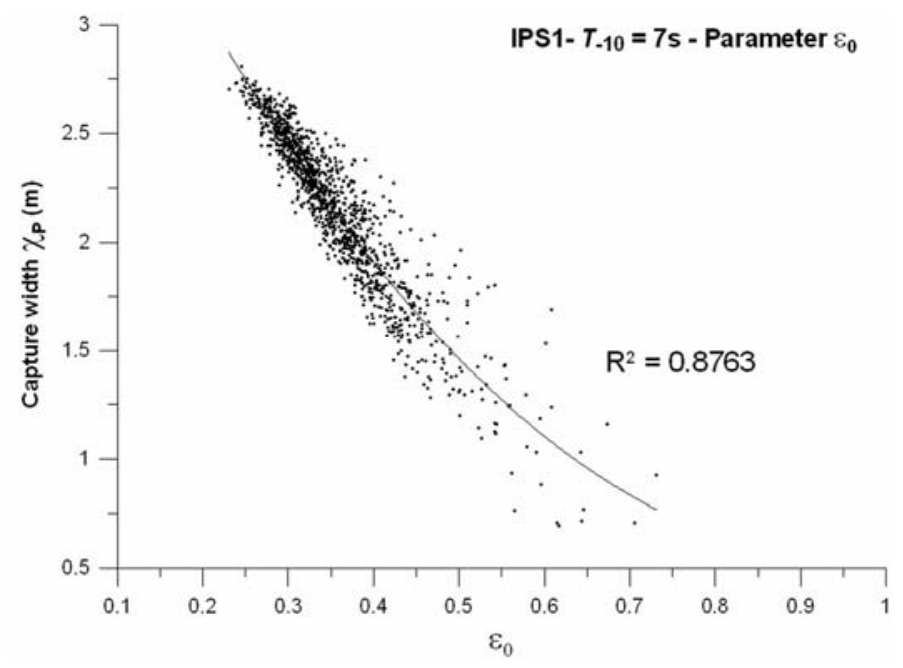

Fig. 8: Distribution of capture width against Mollison's relative bandwidth parameter $\varepsilon_{0}$ in $7 \mathrm{~s}$ energy period sea states in Figueira da Foz for PTO configuration IPS1; quadratic regression in the least-squares sense with determination coefficient $R^{2}$ 

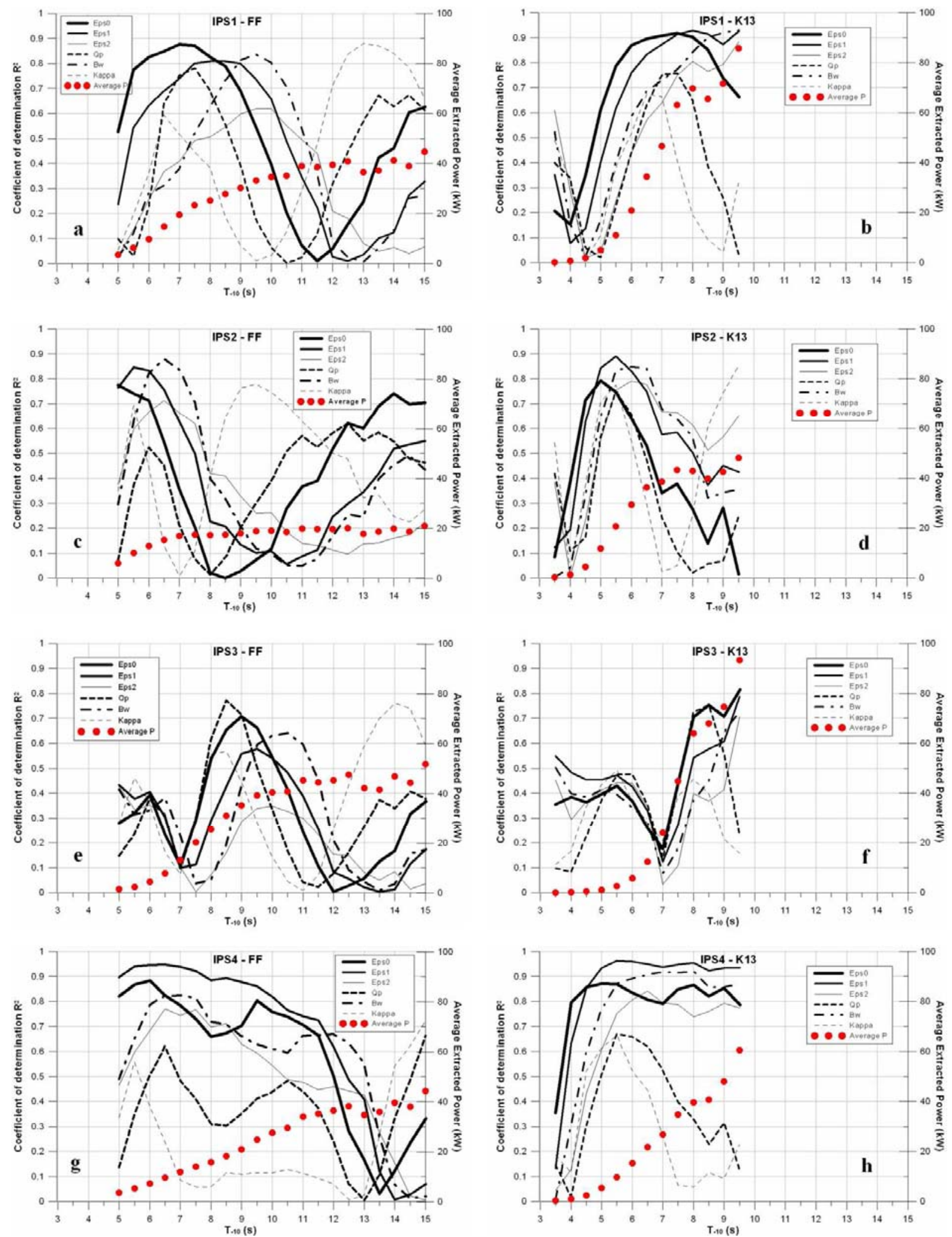

Fig. 9: Determination coefficient $R^{2}$ of quadratic regressions (see Fig. 8) against energy period observed from long-term measurements in Figueira da Foz (left) and K13 (right) for several bandwidth parameters and for PTO configurations IPS1 to IPS4 (top to bottom); mean extracted power by the devices among each sea state sample (spots, right axis) 

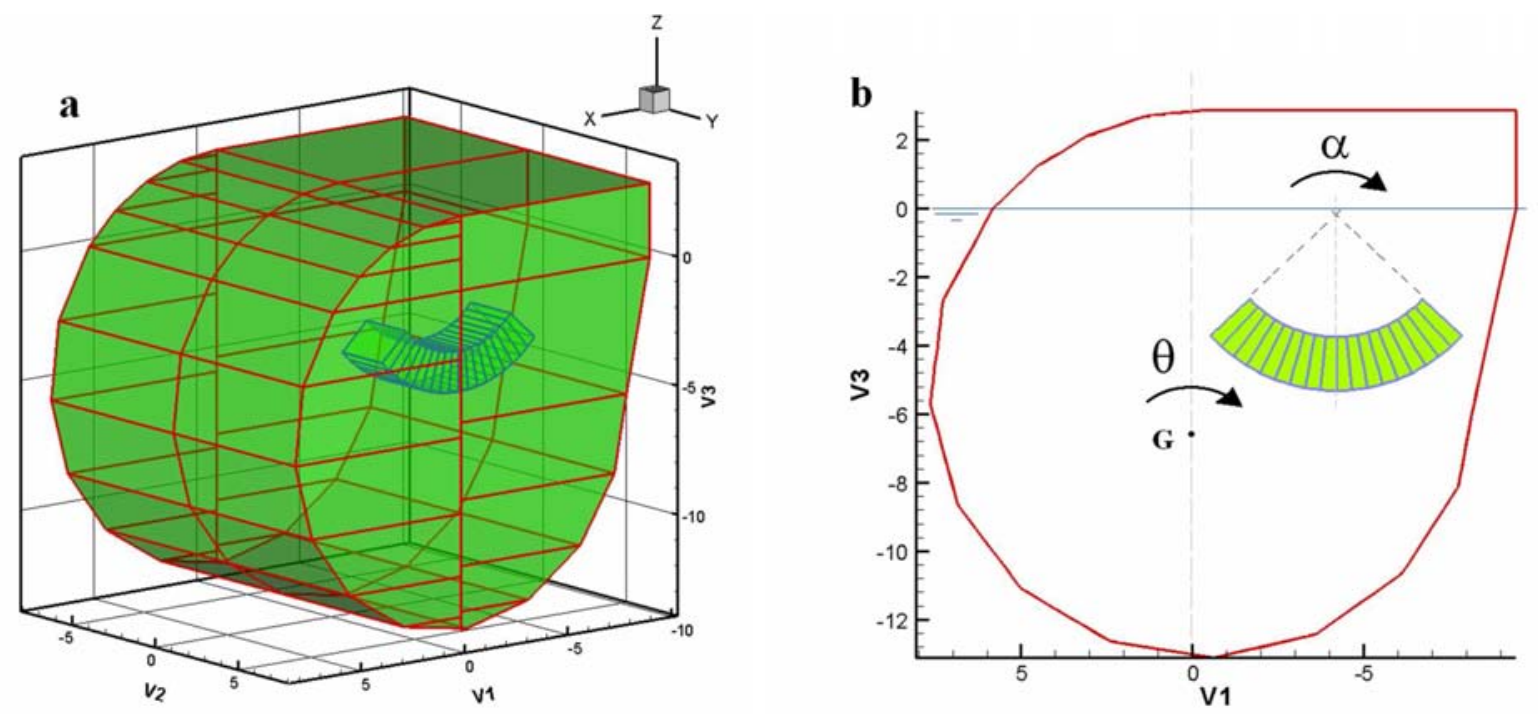

Fig. 10: DES1129 hull of the SEAREV WEC : 3-D view with inner pendulum (a) and sideview physical sketch (b) where $\theta$ and $\alpha$ respectively denote hull's pitch and pendulum's rotation angle (the body is approximately included in a $15 \mathrm{~m}$ side-length cube). 
Click here to download Figure: Fig_11_pdf.pdf

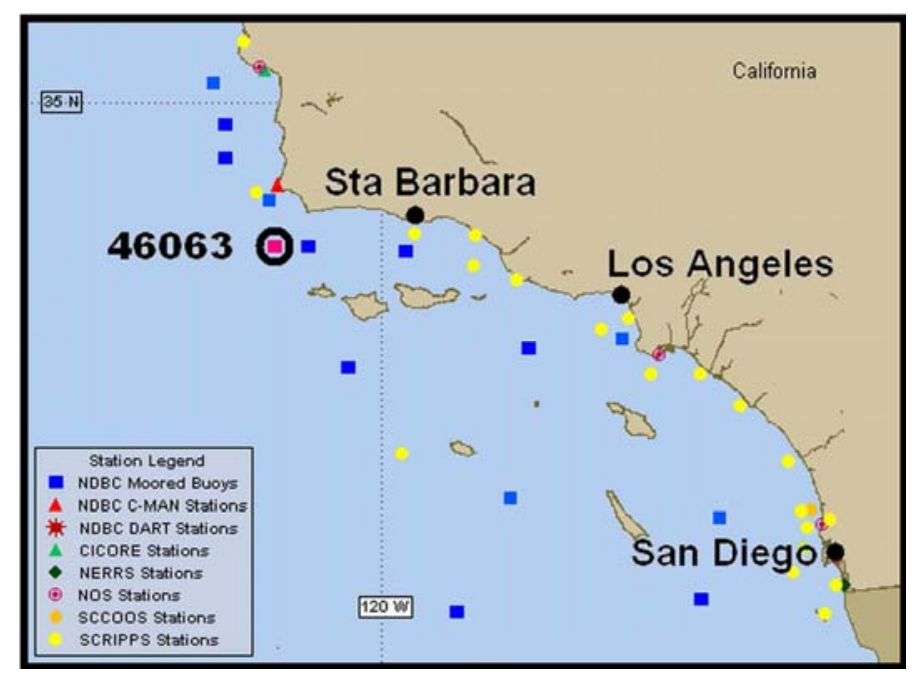

Fig. 11: Californian coast near Los Angeles (USA) : location of NDBC buoy 46063 (Point Conception) [map drawn from NDBC website] 


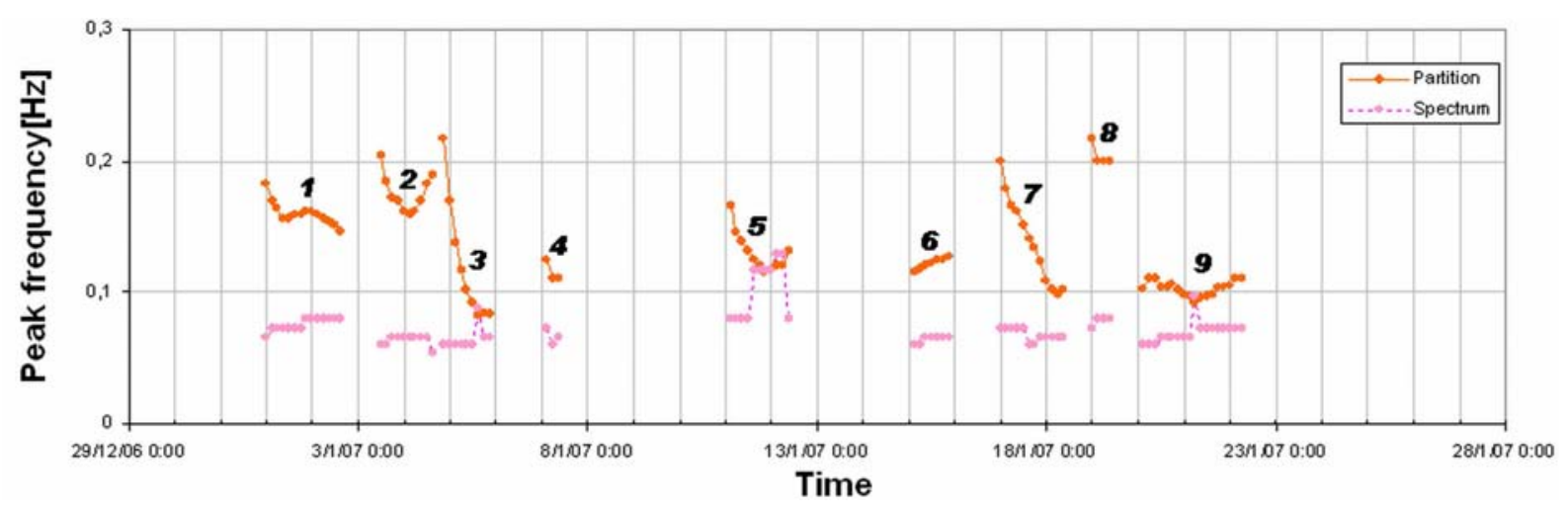

Fig. 12: Peak frequency of active wave system partition and whole spectrum at buoy station NDBC 46063 (January 2007, WW3 data) in nine weather sequences 

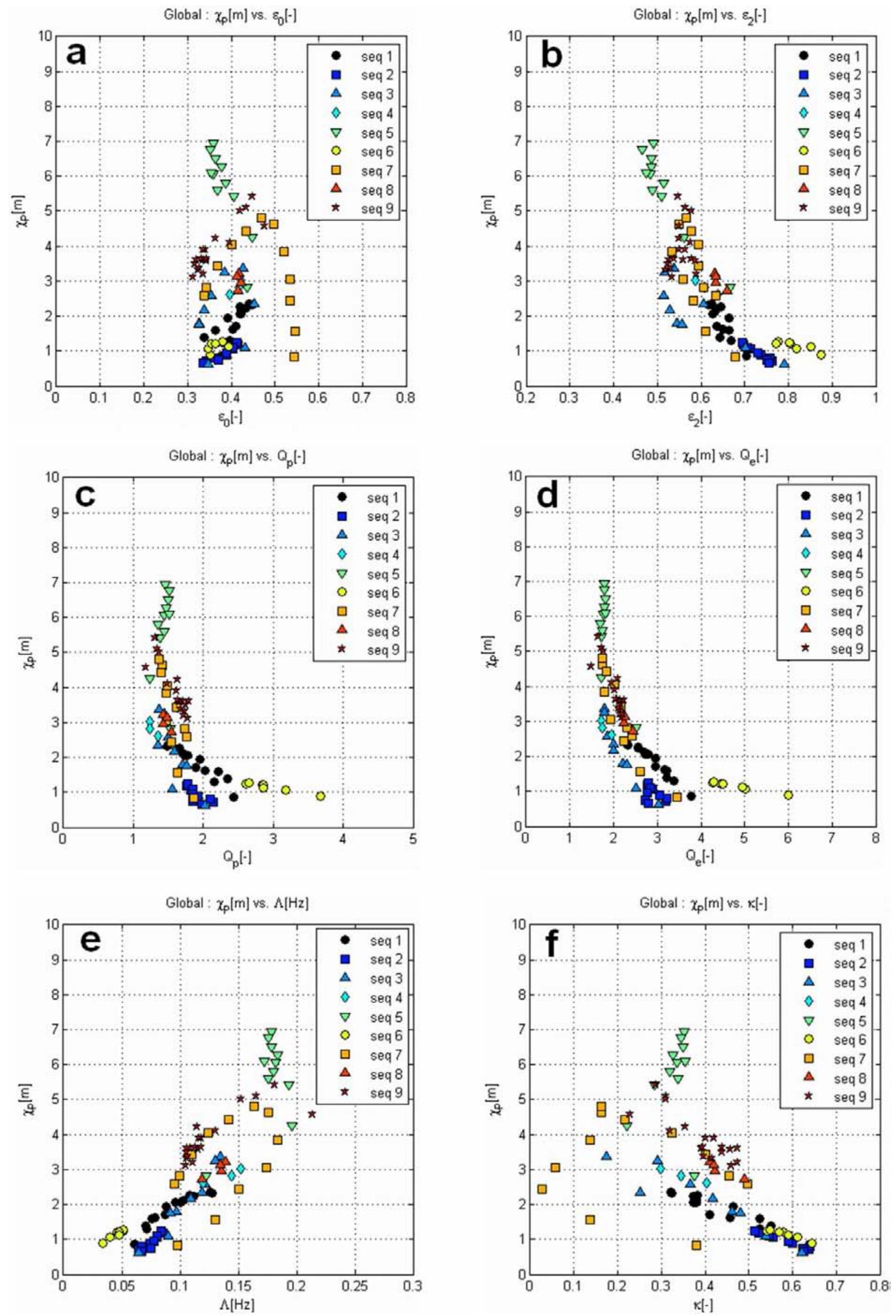

Fig. 13: Scatter plots of SEAREV's capture width obtained in nine sequences (Fig. 12) against several bandwidth parameters (a to f) at station NDBC 46063 (January 2007) 


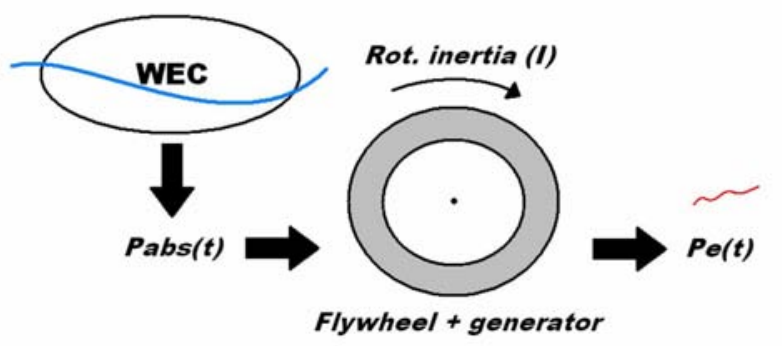

Fig. 14: Simplified sketch of an inertial WEC equipped with flywheel and electrical generator 

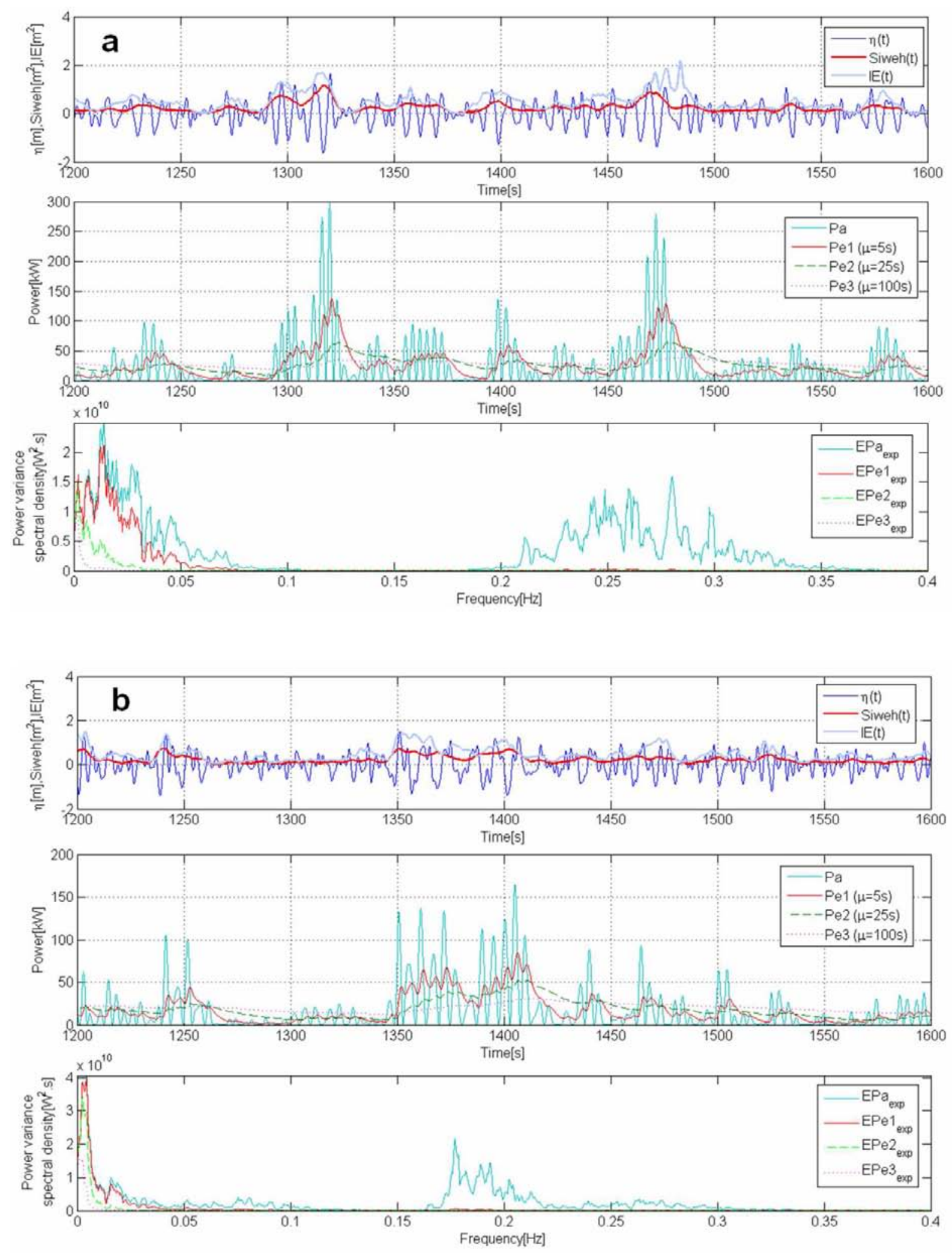

Fig. 15: Simulation of WEC equipped with flywheel in unimodal (a) and bimodal (b) target sea states: wave record and related wave energy histories SIWEH and IE (top), instantaneous power extracted by the WEC $(\mu=0,5,25$ and 100s, middle), related power variance spectra (bottom) 


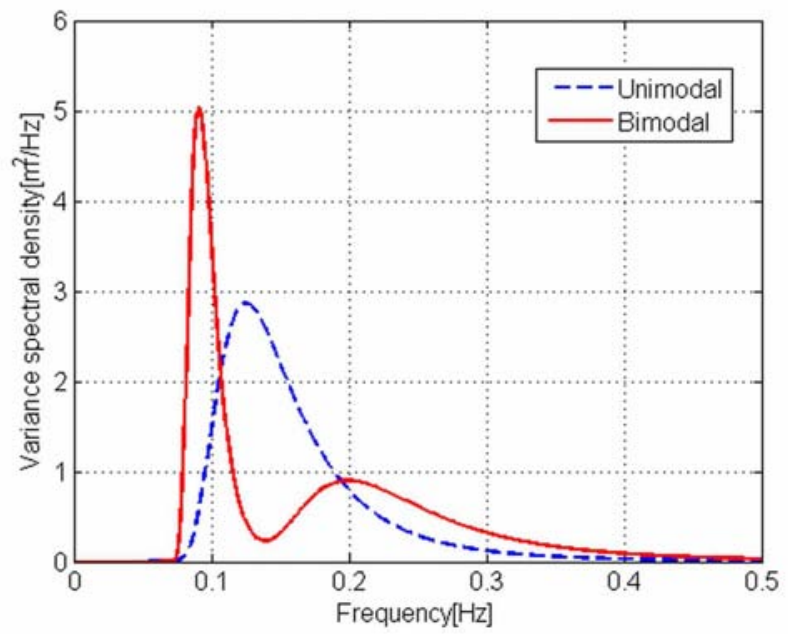

Fig. 16: Unimodal and bimodal target sea states $\left(H_{\mathrm{m} 0}=2 \mathrm{~m}\right.$ and $\left.T-10 \sim 7 \mathrm{~s}\right)$ used for the simulations in Fig. 15 

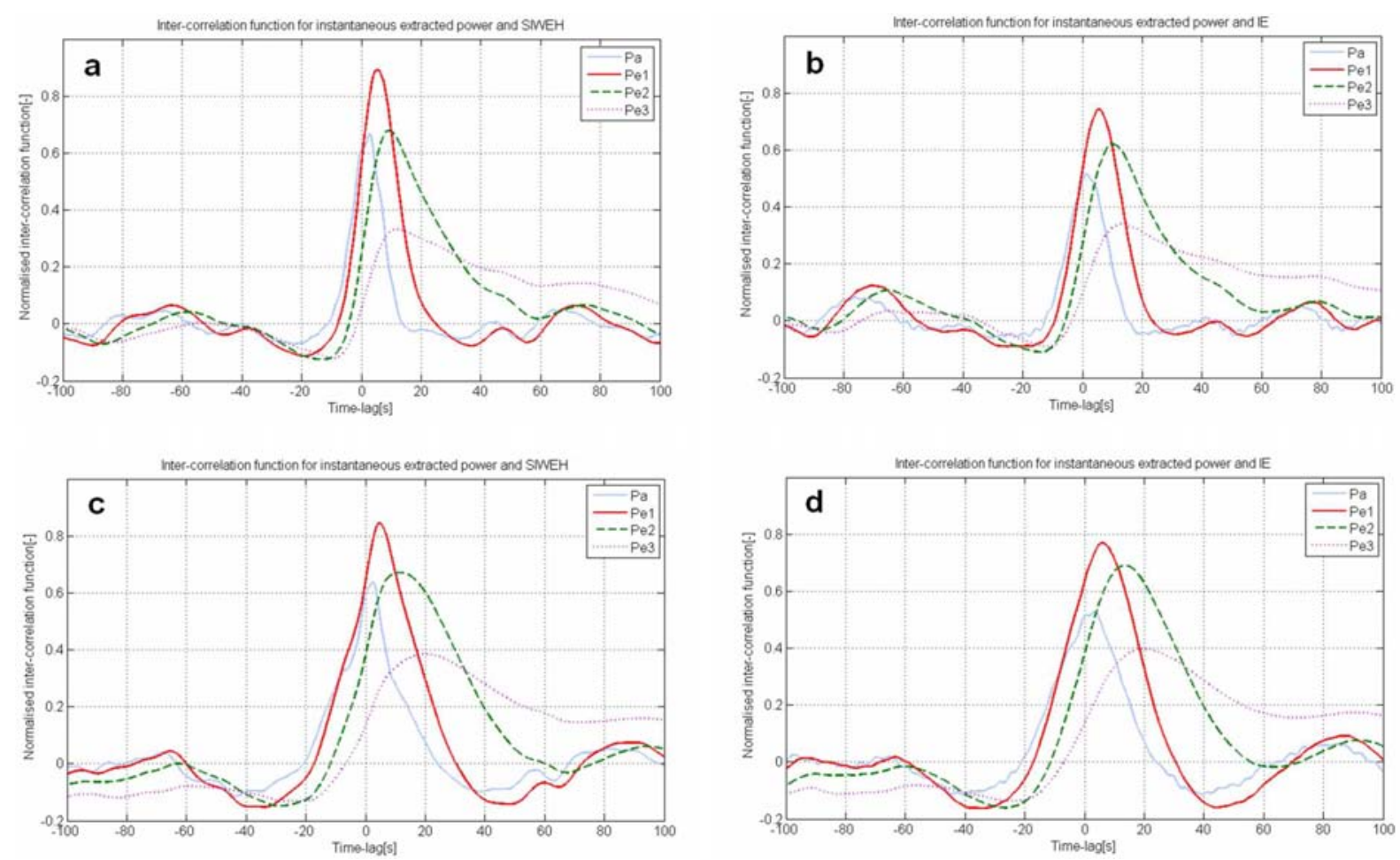

Fig. 17: Intercorrelation function of (normalized and centred) instantaneous power signals output from the flywheel with instantaneous wave energy histories SIWEH (a\&c) and IE (b\&d) in unimodal (top) and bimodal (bottom) sea states (Fig. 16) 


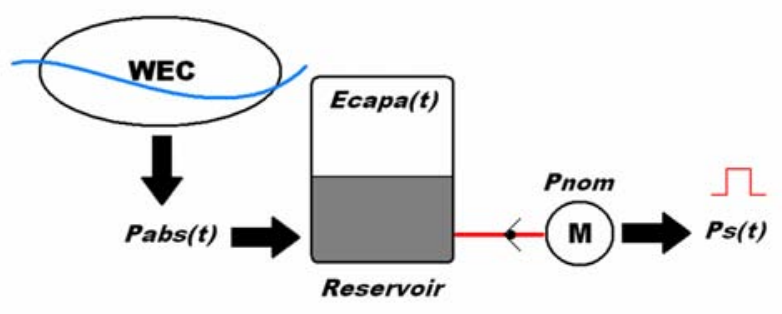

Fig. 18: Simplified sketch of an inertial WEC equipped with short-term energy reservoir and hydraulic motor with nominal power 

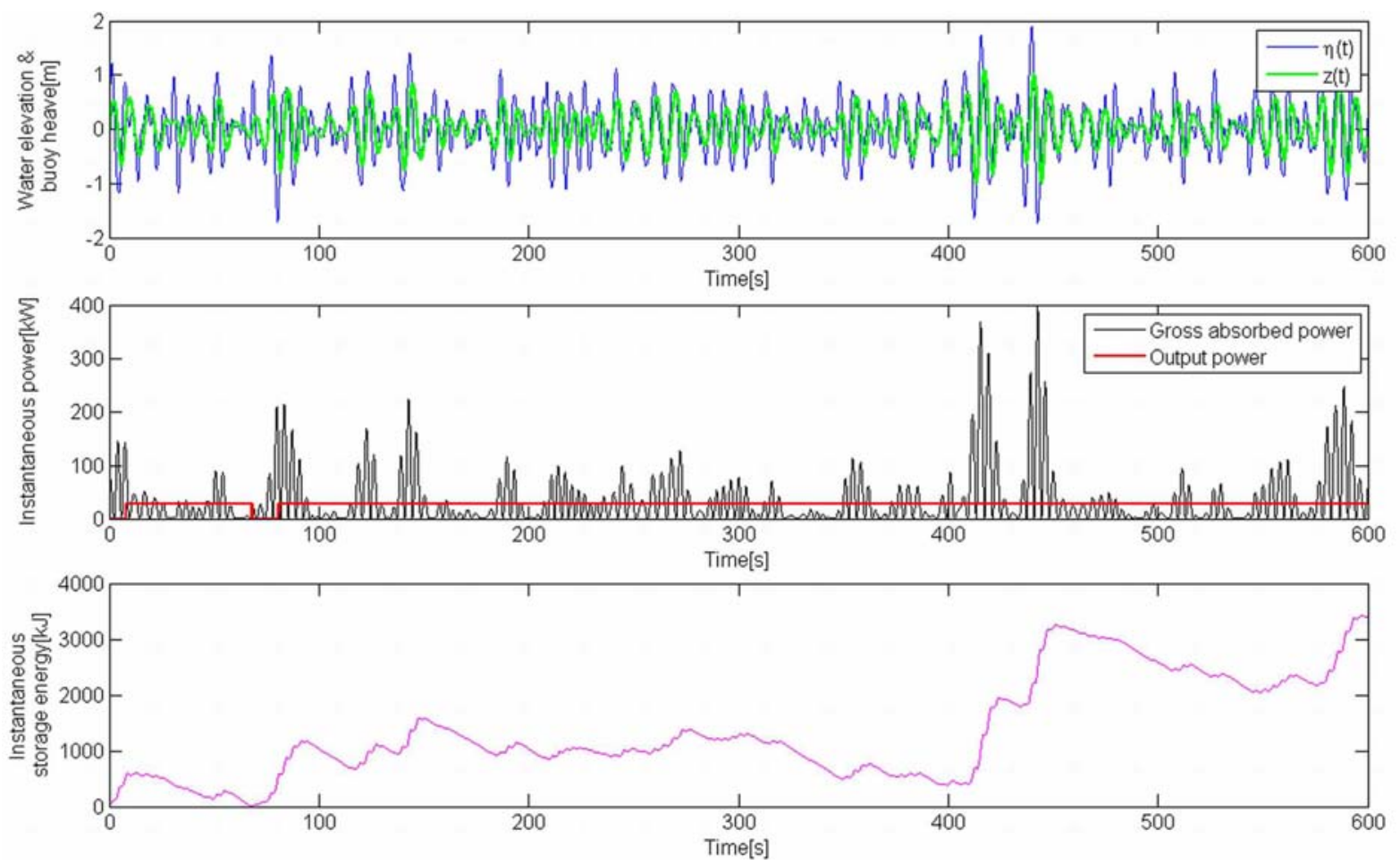

Fig. 19: Simulation of WEC equipped with short-term energy reservoir in unimodal target sea state (Fig. 16): wave record and buoy's heave motions (top), instantaneous power extracted by the WEC with and without reservoir (middle), and instantaneous stored energy inside the reservoir (bottom) 


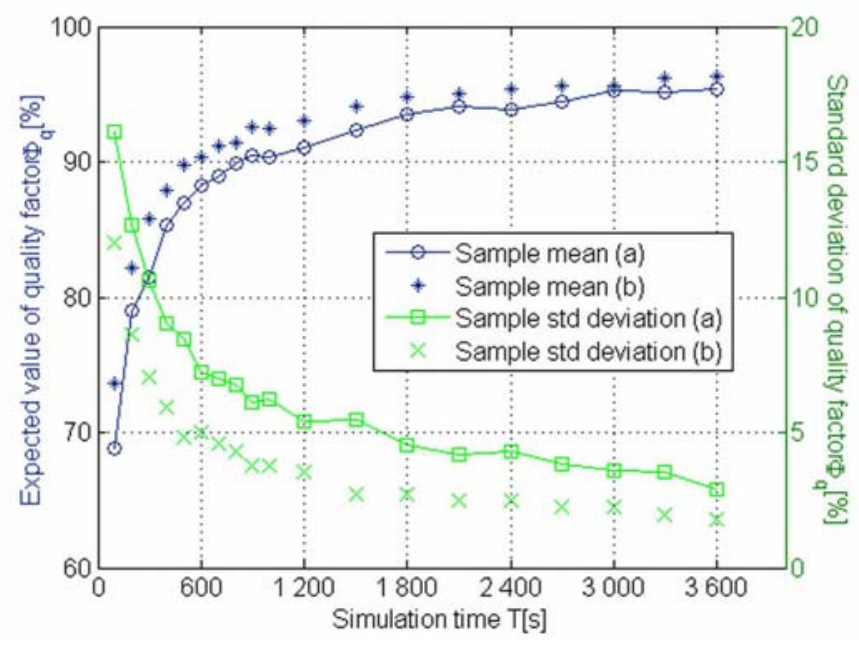

Fig. 20: Expectation (left axis) and standard deviation (right axis) of quality factor $\Phi_{\mathrm{q}}$ against simulation length by simulating waves with random Fourier coefficients $(\operatorname{method} a)$ and random phases only (method $b$ ) 

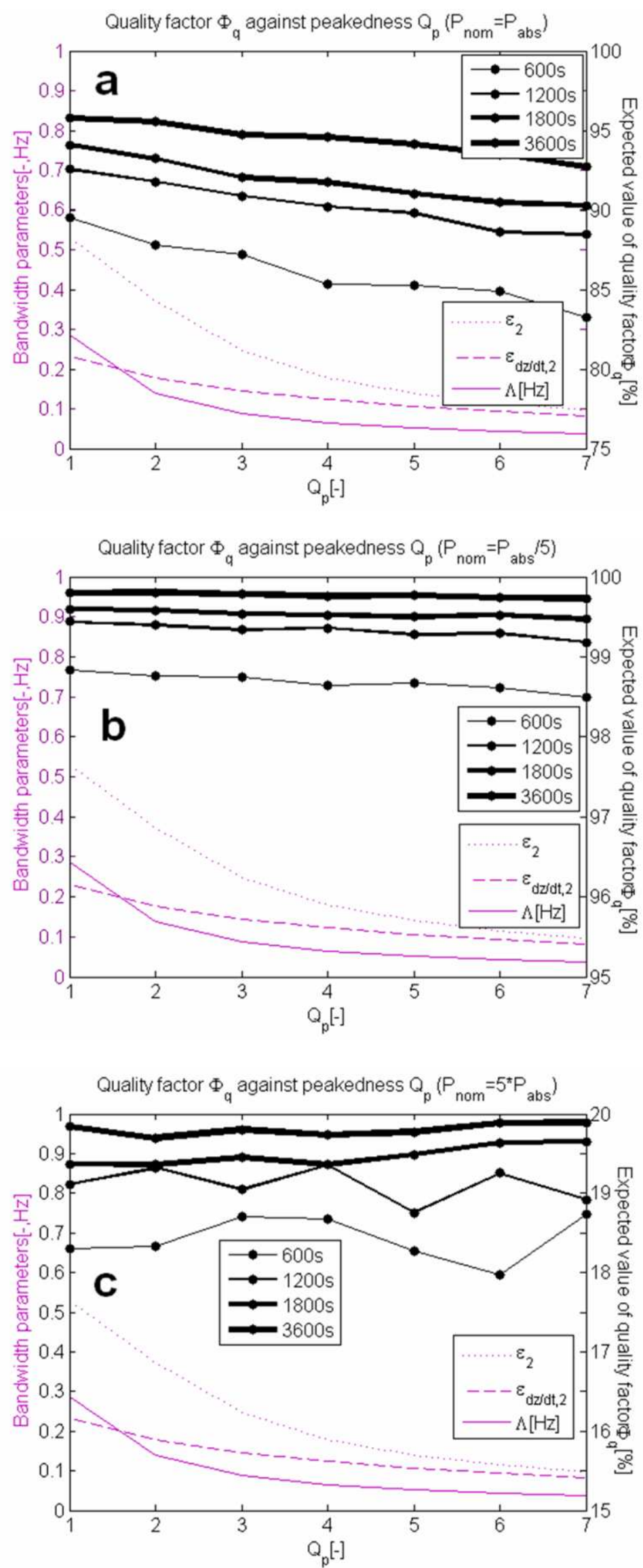

Fig. 21: Expectation of quality factor against peakedness factor $\mathrm{Q}_{\mathrm{p}}$ (unimodal sea states) for several simulation lengths (600 to 3600s) and three operating situations: nominal case $P_{\text {nom }}=P_{\mathrm{a}, \mathrm{m}}(\mathrm{a})$, saturation case $P_{\mathrm{nom}}=P_{\mathrm{a}, \mathrm{m}} / 5$ (b) and underload case $P_{\mathrm{nom}}=P_{\mathrm{a}, \mathrm{m}} * 5$ (c); spectral bandwidth parameters (wave and motions) 


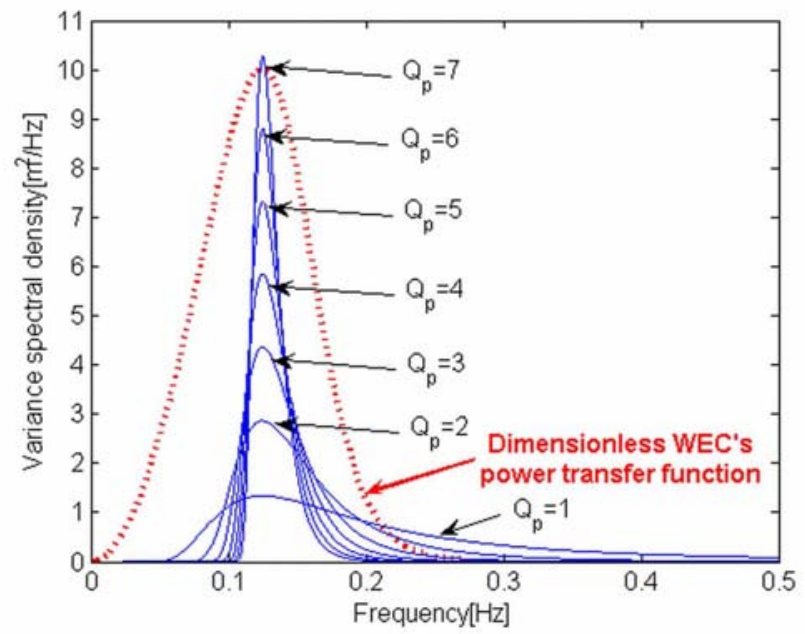

Fig. 22: Target unimodal variance spectral densities with modulable peakedness factor $\left(Q_{\mathrm{p}}=1\right.$ to 7$)$ used in Fig. 21; dimensionless WEC's power transfer function 